MATHEMATICS OF COMPUTATION

Volume 73, Number 248, Pages 1739-1762

S $0025-5718(03) 01616-8$

Article electronically published on August 26, 2003

\title{
A NEW APPROXIMATION TECHNIQUE FOR DIV-CURL SYSTEMS
}

\author{
JAMES H. BRAMBLE AND JOSEPH E. PASCIAK
}

\begin{abstract}
In this paper, we describe an approximation technique for divcurl systems based in $\left(L^{2}(\Omega)^{3}\right)$ where $\Omega$ is a domain in $\mathbb{R}^{3}$. We formulate this problem as a general variational problem with different test and trial spaces. The analysis requires the verification of an appropriate inf-sup condition. This results in a very weak formulation where the solution space is $\left(L^{2}(\Omega)\right)^{3}$ and the data reside in various negative norm spaces. Subsequently, we consider finite element approximations based on this weak formulation. The main approach of this paper involves the development of "stable pairs" of discrete test and trial spaces. With this approach, we enlarge the test space so that the discrete inf-sup condition holds and we use a negative-norm least-squares formulation to reduce to a uniquely solvable linear system. This leads to optimal order estimates for problems with minimal regularity which is important since it is possible to construct magnetostatic field problems whose solutions have low Sobolev regularity (e.g., $\left(H^{s}(\Omega)\right)^{3}$ with $0<s<1 / 2$ ). The resulting algebraic equations are symmetric, positive definite and well conditioned. A second approach using a smaller test space which adds terms to the form for stabilization will also be mentioned. Some numerical results are also presented.
\end{abstract}

\section{INTRODUCTION}

In this paper, we develop a new discretization technique for div-curl systems on a domain $\Omega$ contained in $\mathbb{R}^{3}$. As a model application, we consider the magnetostatic field problem defined by:

$$
\begin{aligned}
\nabla \times \boldsymbol{h} & =\boldsymbol{j} \text { in } \Omega, \\
\nabla \cdot \boldsymbol{b} & =0 \text { in } \Omega, \\
\boldsymbol{b} \cdot \boldsymbol{n} & =0 \text { on } \Gamma, \text { or } \\
\boldsymbol{h} \times \boldsymbol{n} & =0 \text { on } \Gamma .
\end{aligned}
$$

Here $\boldsymbol{b}$ is the magnetic inductance, $\boldsymbol{h}$ is the magnetic field, $\boldsymbol{j}$ is the imposed current and $\boldsymbol{n}$ is the outward normal on $\Gamma$. In the sequel, we shall refer to the above with boundary condition $\boldsymbol{b} \cdot \boldsymbol{n}=0$ as Problem 1 and $\boldsymbol{h} \times \boldsymbol{n}=0$ as Problem 2 .

These problems are augmented with the constitutive relation $\boldsymbol{b}=\mu \boldsymbol{h}$. In many physical applications, $\mu=\mu(x)$ is a nonlinear function (exhibiting hysteresis) depending on $|\boldsymbol{b}(x)|$. However, in this paper, we shall only consider the case when $\mu$ is

Received by the editor January 8, 2003 and, in revised form, March 18, 2003.

2000 Mathematics Subject Classification. Primary 65F10, 65N55.

Key words and phrases. Div-curl systems, inf-sup condition, finite element approximation, Petrov-Galerkin, negative-norm least-squares, Maxwell's equations.

This work was supported in part by the National Science Foundation through grants DMS9805590 and DDS-9973328. 
a fixed given piecewise smooth function. In the presence of material discontinuities (jumps in $\mu$ ), the continuity properties of the fields are given by

$$
[\boldsymbol{h} \times \boldsymbol{n}]=0 \quad \text { and } \quad[\boldsymbol{b} \cdot \boldsymbol{n}]=0
$$

on an interface between materials with normal $\boldsymbol{n}$. Here [.] denotes the jump across the interface. The boundary condition $\boldsymbol{b} \cdot \boldsymbol{n}=0$ corresponds to the boundary of a perfect conductor while $\boldsymbol{h} \times \boldsymbol{n}=0$ corresponds to a magnetic symmetry wall [14].

The above problem is a model in the sense that it is the first (and perhaps simplest) of a series of problems resulting from Maxwell's equations. Some other problems of interest include eddy current problems, driven cavity problems and time-harmonic problems (see, e.g., 4 4 ). Extensions of the new approach developed in this paper to those problems will be the subject of subsequent work.

There have been many approaches for approximating div-curl problems. Nicolaides 23 gave a direct discretization of planar div-curl problems. Later Nicolaides and $\mathrm{Wu} 24$ presented a cofinite volume method for three dimensional div-curl problems. These methods are complicated and quite special. Another approach is a least-squares discretization where the equations are posed in $L^{2}(\Omega)$ [ 8 . The methods proposed require the use of finite elements which are continuous and boundary conditions have to be satisfied. Only two dimensional problems are considered and a high degree of smoothness for the solution is needed in order to prove error estimates. As is well known, (cf. [13]) the solution will be singular at the boundary if the domain is a nonconvex polyhedral domain.

A classical approach for solving the magnetostatic problem is to introduce a scalar or vector potential [4]. For example, one might use $\boldsymbol{A}$ satisfying $\boldsymbol{B}=\nabla \times \boldsymbol{A}$. This gives rise to a problem on a subspace $\boldsymbol{M}$ of $\boldsymbol{H}$ (curl) satisfying appropriate boundary conditions. Here $\boldsymbol{H}$ (curl) is the set of functions, which along with their curl, are in $\left(L^{2}(\Omega)\right)^{3}$. Specifically, one gets the variational problem: Find $\boldsymbol{A} \in \boldsymbol{M}$ satisfying

$$
\left(\mu^{-1} \nabla \times \boldsymbol{A}, \nabla \times \phi\right)=(\boldsymbol{j}, \boldsymbol{\phi}) \quad \text { for all } \boldsymbol{\phi} \in \boldsymbol{M},
$$

augmented with a "gauge condition" such as $\nabla \cdot \boldsymbol{A}=0$ in $\Omega$. This gives rise to a mixed system when one introduces a pressure $P$ (which is identically zero).

Many authors have pointed out the problems of applying standard conforming $H^{1}$ finite elements to the above problem (see, e.g., [4], 10, 11], 26]). In fact, in [11], it was shown that the direct application of conforming $H^{1}$ finite elements to problems in $\boldsymbol{H}$ (curl) can lead to discrete solutions which fail to converge to the solution as the mesh size tends to zero. Techniques for fixing up the Galerkin method by introducing weighted approximations and boundary penalty terms have been proposed in 11], 12. A discontinuous Galerkin method for the time-harmonic Maxwell equations was introduced in [26]. Their aim was to develop a flexible scheme in which the order of the scheme can change between different regions of the grid and standard elements can be used.

Alternatively, mixed finite element approaches have been extensively studied (see [4], [7, 14], 15, 26] and the included references). These are based on the curl conforming approximation spaces introduced by Nedelec [21, [22] and result in discrete saddle point systems. Higher order Nedelec elements are quite complicated. The solution methods for the resulting algebraic problems have only been recently developed (see, e.g., [20], [19], [18], [16], [27], [28]). Together with the use of the above cited techniques for the solution of the resulting algebraic equations, the edge 
element approach can be quite effective, especially for lower order elements. In some mixed formulations, the quantity of interest $\boldsymbol{B}$ is obtained by differentiation of the potential and results in a discontinuous approximation. In contrast, the actual solution is smooth away from the interfaces.

In this paper, we propose a new variational formulation of the above problem along with a new discretization technique for its approximation. Our approach has the following properties: First, we directly approximate the variables of interest (either $\boldsymbol{h}$ or $\boldsymbol{b}$ ). Potentials and the resulting differentiation of approximate solutions are avoided. The method is based in $\left(L^{2}(\Omega)\right)^{3}$ and so duality techniques are avoided. The scheme is convergent for the type of problems with low regularity which appear in practical applications. The discretization allows for the mixing of continuous and discontinuous approximation spaces of varying polynomial degrees on the underlying mesh partitioning. This enables the use of higher order (continuous) elements away from the interface where the solution is smooth coupled with low order discontinuous elements near the interfaces. Our approach may be viewed as a negative-norm least-squares method. Negative-norm least-squares methods for elliptic problems have been proposed and developed, for example, in [5] and [6].

The solution of the discrete equations which result from the method to be developed can be efficiently computed. In fact, the formulation utilizes preconditioners for standard second order problems. The resulting discrete system is uniformly equivalent, independent of the mesh size, to the mass matrix. Even in the case of mesh refinement, the mass matrix can be preconditioned by its diagonal.

We should remark that there have been other studies of div-curl solutions based on $L^{2}(\Omega)$. For example, Auchmuty and Alexander [2] prove $\left(L^{2}(\Omega)\right)^{2}$ solutions of two dimensional div-curl systems under the assumption of $C^{1,1}$ boundaries in the presence of mixed boundary conditions and data in $L^{p}(\Omega)$ for $p>1$. Our assumptions on the data are much weaker although we do not consider the case of multiply connected domains with mixed boundary conditions.

An outline of the paper is as follows. In Section 2 we present an abstract variational problem, which will be the basis for the treatment of our problems. In Section 3 we give some preliminary results needed for our treatment of the div-curl problems. Weak variational formulations of Problems 1 and 2 are presented in Sections 4 and 5, respectively. In Section 6 we provide a construction of stable pairs of finite element spaces which can be used for the approximate solutions of these problems. Finally we give the results of some numerical computations in Section 7 .

\section{General variational and Least-Squares dual Formulation}

In this section, we consider general variational problems on Hilbert spaces. We start with a Lax-Milgram theorem. Next, by modifying the space of test functions, we develop a second variational formulation related to least-squares in a dual norm. Although some of our results here are classical, they set the stage for the approximations to div-curl systems to be developed in the subsequent sections.

Let $X$ and $Y$ be two Hilbert Spaces. We shall use $(u, v)_{X},(u, v)_{Y},\|u\|_{X}$ and $\|u\|_{Y}$ to denote the corresponding inner products and norms. Let $X^{*}$ and $Y^{*}$ denote the dual spaces (the spaces of bounded linear functionals on $X$ and $Y$, respectively). Since $X$ and $Y$ are Hilbert spaces, $X^{*}$ and $Y^{*}$ are also. The dual norm on $X^{*}$ is 
defined by

$$
\|l\|_{X^{*}}=\sup _{x \in X} \frac{\langle l, x\rangle}{\|x\|_{X}}
$$

Here $\langle l, x\rangle$ denotes the value of the functional $l$ at $x$. Let $T_{X}: X^{*} \rightarrow X$ be the operator defined by

$$
\left(T_{X} l, x\right)_{X}=\langle l, x\rangle \quad \text { for all } x \in X .
$$

Then it is easy to see that

$$
(l, \tilde{l})_{X^{*}}=\left\langle l, T_{X} \tilde{l}\right\rangle \quad \text { for all } l, \tilde{l} \in X^{*} .
$$

The analogous definitions and identities hold for the dual space $Y^{*}$.

We start with a generalized Lax-Milgram theorem. Suppose that $b(\cdot, \cdot)$ is a continuous bilinear form (with bound $\|b\|$ ) on $X \times Y$ satisfying the "inf-sup" condition

$$
\|x\|_{X} \leq C_{0} \sup _{y \in Y} \frac{b(x, y)}{\|y\|_{Y}}, \quad \text { for all } x \in X .
$$

Let $Y_{0}=\{y \in Y: b(x, y)=0$ for all $x \in X\}$. We then have the following theorem which is essentially contained in [3]:

Theorem 2.1 (Lax-Milgram). Let $F$ be in $Y^{*}$ and let $b(\cdot, \cdot)$ be as above. Then there exists a unique $x \in X$ satisfying

$$
b(x, y)=\langle F, y\rangle \quad \text { for all } y \in Y
$$

if and only if $\langle F, y\rangle=0$ for all $y \in Y_{0}$. Furthermore, if the solution $x$ to (2.2) exists, then it satisfies

$$
\|x\|_{X} \leq C_{0}\|F\|_{Y^{*}}
$$

Now if $\langle F, y\rangle=0$ for all $y \in Y_{0}$, then (2.2) is the same as

$$
b(x, y)=\langle F, y\rangle \quad \text { for all } y \in Y_{1},
$$

where $Y_{1}$ is the orthogonal complement of $Y_{0}$ in $Y$. Let $B: X \rightarrow Y^{*}$ be defined by

$$
\langle B x, y\rangle=b(x, y) \text { for all } x \in X, y \in Y .
$$

Then it is easy to see that $Y_{1}=\left\{y \in Y: y=T_{Y} B x\right.$ for $\left.x \in X\right\}$. Thus it follows immediately that by (2.1)

$$
\|x\|_{X} \leq C_{0} \sup _{y \in Y} \frac{b(x, y)}{\|y\|_{Y}}=C_{0} \sup _{y \in Y_{1}} \frac{b(x, y)}{\|y\|_{Y}}=C_{0}\|B x\|_{Y^{*}},
$$

i.e., the inf-sup condition holds for $X \times Y_{1}$.

The following theorem is an immediate consequence of the above arguments.

Theorem 2.2. Let $F$ be in $Y_{1}^{*}$ and let $b(\cdot, \cdot)$ be as above. Then if (2.1) holds, there exists a unique function $x \in X$ satisfying (2.3). Furthermore, if (2.2) has a solution, then the solution of (2.2) and (2.3) coincide.

Remark 2.1. The formulation (2.3) is a least-squares variational formulation based in the dual space $Y^{*}$. Indeed, the solution $x$ of (2.3) satisfies

$$
A(x, \tilde{x}) \equiv(B x, B \tilde{x})_{Y^{*}}=(F, B \tilde{x})_{Y^{*}} \quad \text { for all } \tilde{x} \in X .
$$

Note that $A$ coerces the square of the norm of $X$ by (2.4) and is bounded. Clearly, $(F, B \tilde{x})_{Y^{*}}$ is a bounded functional on $X$.

Remark 2.2. The two formulations are obviously not equivalent as the least-square formulation (2.3) produces a solution even when the solution of (2.2) does not exist. 
We next consider approximation. Specifically, suppose that we have a family of "discrete" subspaces $\left\{X_{h} \subseteq X\right\}$ and $\left\{Y_{h} \subseteq Y\right\}$ for $h \in(0,1]$. Here we think of $h$ as an approximation parameter and $X_{h}$ as an approximation to $X$. In all of our applications, $Y_{h}$ is an approximation space to $Y$ although this is not necessary in the subsequent development. We assume that the pair $\left(X_{h}, Y_{h}\right)$ satisfies the discrete inf-sup condition,

$$
\|x\|_{X} \leq C_{1} \sup _{y \in Y_{h}} \frac{b(x, y)}{\|y\|_{Y}}, \quad \text { for all } x \in X_{h},
$$

with constant $C_{1}$ independent of $h$.

We suppose that $F$ satisfies the compatiblity conditions so that (2.2) has a unique solution. Note that, in general, $F$ does not satisfy the discrete compatibility condition, i.e, if $y \in Y_{h}$ satisfies $b(x, y)=0$ for all $x \in X_{h}$, it does not follow that $\langle F, y\rangle=0$. Thus, the discrete problem: find $x \in X_{h}$ satisfying

$$
b(x, y)=\langle F, y\rangle \text { for all } y \in Y_{h},
$$

does not have a solution, in general. Nevertheless we can define the least-squares approximation to (2.2) by applying the above constructions. Specifically, we define $B_{h}: X_{h} \rightarrow Y_{h}^{*}$ by

$$
\left\langle B_{h} x, y\right\rangle=b(x, y), \quad \text { for all } x \in X_{h}, y \in Y_{h} .
$$

The approximate solution $x_{h}$ is the unique element of $X_{h}$ satisfying

$$
A_{h}\left(x_{h}, x\right) \equiv\left\langle B_{h} x_{h}, T_{Y_{h}} B_{h} x\right\rangle=\left\langle F, T_{Y_{h}} B_{h} x\right\rangle, \quad \text { for all } x \in X_{h} .
$$

As in the continuous case, for $z_{h} \in X_{h}$,

$$
\sup _{y \in Y_{h}} \frac{b\left(z_{h}, y\right)}{\|y\|_{Y}}=\left\langle B_{h} z_{h}, T_{Y_{h}} B_{h} z_{h}\right\rangle^{1 / 2} .
$$

The following theorem shows that $x_{h}$ is a quasi-optimal approximation to the solution of (2.2).

Theorem 2.3. Assume that $F$ satisfies the assumptions of Theorem 2.1 and that (2.2) has a unique solution $x$. Assume that (2.6) is satisfied and let $x_{h}$ solve (2.7). Then

$$
\left\|x-x_{h}\right\|_{X} \leq\left(1+C_{1}^{2}\|b\|^{2}\right) \inf _{\zeta \in X_{h}}\|x-\zeta\|_{X}
$$

Proof. Let $v$ be in $X_{h}$ and set $z=T_{Y_{h}} B_{h} v$. Then

$$
b\left(x_{h}, z\right)=\langle F, z\rangle=b(x, z) .
$$

Using (2.8) and (2.6), for any $\zeta \in X_{h}$,

$$
\begin{aligned}
\left\|x_{h}-\zeta\right\|_{X}^{2} & \leq C_{1}^{2}\left\langle B_{h}\left(x_{h}-\zeta\right), T_{Y_{h}} B_{h}\left(x_{h}-\zeta\right)\right\rangle \\
& =C_{1}^{2} b\left(x_{h}-\zeta, T_{Y_{h}} B_{h}\left(x_{h}-\zeta\right)\right) \\
& =C_{1}^{2} b\left(x-\zeta, T_{Y_{h}} B_{h}\left(x_{h}-\zeta\right)\right) \\
& \leq C_{1}^{2}\|b\|^{2}\|x-\zeta\|_{X}\left\|x_{h}-\zeta\right\|_{X} .
\end{aligned}
$$

Thus,

$$
\left\|x-x_{h}\right\|_{X} \leq\|x-\zeta\|_{X}+\left\|\zeta-x_{h}\right\|_{X} \leq\left(1+C_{1}^{2}\|b\|^{2}\right)\|x-\zeta\|_{X} .
$$

This completes the proof of the theorem. 
In applications, it is sometimes more efficient to replace the operator $T_{Y_{h}}$ by a preconditioner $\widetilde{T}_{Y_{h}}: Y_{h}^{*} \rightarrow Y_{h}$. In the div-curl application discussed in the remainder of this paper, $T_{Y_{h}}$ involves multiple applications of the inverse of the discrete Laplacian. The development of preconditioners for second order problems has been extensively researched (see, for example, the proceedings of the series of the International Conferences on Domain Decomposition, http://www.ddm.org). The most effective techniques based on multigrid and domain decomposition are implemented in many existing software packages, e.g., PETSc $(\mathrm{http} / /$ www-unix.mcs.anl.gov/ petsc/petsc-2/) or hypre (http://www.llnl.gov/CASC/hypre/).

We have the following corollary which follows easily from the above proof.

Corollary 2.1. Assume that the hypotheses of Theorem 2.3 are satisfied and, in addition, that there are constants $a_{0}>0$ and $a_{1}$ not depending on $h$ and satisfying

$$
a_{0}\left\langle G, \widetilde{T}_{Y_{h}} G\right\rangle \leq\left\langle G, T_{Y_{h}} G\right\rangle \leq a_{1}\left\langle G, \widetilde{T}_{Y_{h}} G\right\rangle, \quad \text { for all } G \in Y_{h}^{*} .
$$

Suppose that $x_{h} \in X_{h}$ satisfies (2.7) with $T_{Y_{h}}$ replaced by $\widetilde{T}_{Y_{h}}$. Then,

$$
\left\|x-x_{h}\right\|_{X} \leq\left(1+\frac{a_{1} C_{1}^{2}\|b\|^{2}}{a_{0}}\right) \inf _{\zeta \in X_{h}}\|x-\zeta\|_{X} .
$$

Remark 2.3. Since $F$ does not satisfy the discrete compatibility conditions, the solution $x_{h}$ may depend on the choice of $\widetilde{T}_{Y_{h}}$. However, if the approximation pair were to satisfy the condition that continuous compatibility implies discrete compatibility, then the resulting solutions would be guaranteed to be independent of $\widetilde{T}_{Y_{h}}$.

In practice, one needs to solve the discrete system: Find $x_{h} \in X_{h}$ satisfying

$$
\widetilde{A}_{h}\left(x_{h}, \tilde{x}_{h}\right) \equiv\left\langle B_{h} x_{h}, \widetilde{T}_{Y_{h}} B_{h} \tilde{x}_{h}\right\rangle=b\left(\tilde{x}_{h}, \widetilde{T}_{Y_{h}} F\right) \text { for all } \tilde{x}_{h} \in X_{h} .
$$

Let $\left\{x_{h}^{i}\right\}$ and $\left\{y_{h}^{i}\right\}$ denote the (local finite element) bases for $X_{h}$ and $Y_{h}$, respectively, and define the matrix $B_{j i}=b\left(x_{h}^{i}, y_{h}^{j}\right)$. The right-hand side of (2.9) is computed by one application each of $B^{t}$ (the transpose of $B$ ) and $\widetilde{T}_{Y_{h}}$. The "stiffness" matrix corresponding to $\widetilde{A}_{h}(\cdot, \cdot)$ is full and one never computes it in practice. Instead, we compute $x_{h}$ by preconditioned iteration. To implement such an iteration, we simply have to compute the action of the stiffness matrix and that of a preconditioner, $\widetilde{T}_{X_{h}}: X_{h}^{*} \rightarrow X_{h}$, once per step in the iteration. Using the identity

$$
\widetilde{A}_{h}\left(x_{h}, x_{h}^{i}\right)=b\left(x_{h}^{i}, \widetilde{T}_{Y_{h}} B_{h} x_{h}\right),
$$

we see that the action of the stiffness matrix reduces to one application each of $B, B^{t}$ and $\widetilde{T}_{Y_{h}}$. We get a uniformly convergent (independent of $h$ ) iteration if the preconditioner $\widetilde{T}_{X_{h}}$ satisfies

$$
b_{0}\left\langle G, \widetilde{T}_{X_{h}} G\right\rangle \leq\left\langle G, T_{X_{h}} G\right\rangle \leq b_{1}\left\langle G, \widetilde{T}_{X_{h}} G\right\rangle, \quad \text { for all } G \in X_{h}^{*}
$$

with $b_{0}, b_{1}$ independent of $h$. In fact, it follows from the above inequalities that the condition number of the preconditioned system is bounded by

$$
\frac{a_{1} b_{1} C_{0}^{2}\|b\|^{2}}{a_{0} b_{0}} \text {. }
$$

Remark 2.4. We note that the system (2.9) is a Petrov-Galerkin approximation based on the bilinear form $b(\cdot, \cdot)$ and the spaces $X_{h}$ and $\widetilde{Z}_{h}=\left\{\widetilde{T}_{Y_{h}} B_{h} \zeta: \zeta \in X_{h}\right\}$. 
Remark 2.5. For our applications, $\|\cdot\|_{X}$ is $\left(L^{2}(\Omega)\right)^{3}$ and $\tilde{T}_{X_{h}}$ can be taken to be a diagonal scaling.

\section{Preliminaries For the DiV-CURL PROBlems}

In this section, we provide some preliminary notation and lemmas concerning spaces and operators. We start with a simply connected bounded polyhedral domain $\Omega$ contained in $\mathbb{R}^{3}$. Its boundary will be denoted by $\Gamma$ and can be written,

$$
\Gamma=\bigcup_{i=0}^{p} \Gamma_{i}
$$

where $\left\{\Gamma_{i}\right\}$ are the connected components of $\Gamma$ and $\Gamma_{0}$ denotes the outer boundary.

We choose to treat here in detail the three dimensional case. Analogous results for the simpler case of $\Omega \subset \mathbb{R}^{2}$ are easily obtained. We indicate this in Section 7 , where we present numerical tests for examples in two dimensions.

We shall have to switch between vector and scalar functions in our exposition. We shall use $(\cdot, \cdot)$ to denote both the inner product in $L^{2}(\Omega)$ and $\boldsymbol{L}^{2}(\Omega)=\left(L^{2}(\Omega)\right)^{3}$. Similarly, $\|\cdot\|$ will denote the norms. We shall always use bold face to indicate vector functions. In this way, there will be no difficulty in determining which of the above norms or inner products are being used.

We shall use the following function spaces:

$$
\begin{aligned}
H_{1} & =H^{1}(\Omega), \\
\boldsymbol{V}_{1} & =\boldsymbol{H}_{0}^{1}(\Omega) \equiv\left(H_{0}^{1}(\Omega)\right)^{3}, \\
\boldsymbol{H}(\operatorname{curl}) & =\left\{\boldsymbol{v} \in \boldsymbol{L}^{2}(\Omega): \nabla \times \boldsymbol{v} \in \boldsymbol{L}^{2}(\Omega)\right\}, \\
\boldsymbol{H}_{0}(\operatorname{curl}) & =\{\boldsymbol{v} \in \boldsymbol{H}(\operatorname{curl}): \boldsymbol{v} \times \boldsymbol{n}=0\}, \\
\boldsymbol{H}(\operatorname{div}) & =\left\{\boldsymbol{v} \in \boldsymbol{L}^{2}(\Omega): \nabla \cdot \boldsymbol{v} \in L^{2}(\Omega)\right\} .
\end{aligned}
$$

Our problems will involve a parameter $\mu$ which corresponds, for example, to the magnetic permeability in a magnetostatic field problem. We shall assume that there are constants $\mu_{0}$ and $\mu_{1}$ satisfying $0<\mu_{0} \leq \mu(x) \leq \mu_{1}$, for all $x \in \Omega$. The norms for all of the spaces, except that for $H_{1}$, in (3.1) are the usual ones; see, e.g., [15]. For functions $\psi \in H_{1}$, we will use the equivalent norm

$$
\|\psi\|_{H_{1}}=\left(\|\nabla \psi\|_{\mu}^{2}+|\bar{\psi}|^{2}\right)^{1 / 2}
$$

where $\bar{\psi}$ is the mean value of $\psi$.

We will also need the following auxiliary functions. For each $i>0$, we define $\psi_{i}$ to be the function in $H^{1}(\Omega)$ satisfying

$$
\begin{aligned}
-\nabla \cdot \mu \nabla \psi_{i} & =0, \quad \text { in } \Omega, \\
\psi_{i} & =\delta_{i j}, \quad \text { on } \Gamma_{j} .
\end{aligned}
$$

Here $\delta_{i j}$ denotes the Kronecker Delta. We define $W_{d}$ to be the span of $\psi_{i}, i=$ $1, \ldots, p$. We now set

$$
\begin{aligned}
& H_{2}=H_{0}^{1}(\Omega) \oplus W_{d} \quad \text { and } \\
& \boldsymbol{V}_{2}=\left(H^{1}(\Omega)\right)^{3} .
\end{aligned}
$$

The following proposition is a slight modification of Corollary 3.4 of [15]. 
Proposition 3.1. Let $\boldsymbol{u}$ be in $\boldsymbol{L}^{2}(\Omega)$. Then there exists a decomposition

$$
\boldsymbol{u}=\nabla \times \boldsymbol{v}+\mu \nabla \psi
$$

where $\boldsymbol{v} \in \boldsymbol{H}_{0}($ curl $)$ and $\psi \in H^{1}(\Omega)$.

We also use the following proposition from [25].

Proposition 3.2. Let $\boldsymbol{v}$ be in $\boldsymbol{H}_{0}(\mathbf{c u r l})$. Then there exists a decomposition

$$
\boldsymbol{v}=\boldsymbol{w}+\nabla \psi
$$

where $\psi \in H^{1}(\Omega)$ and $\boldsymbol{w} \in \boldsymbol{V}_{1}$ satisfies

$$
\|\boldsymbol{w}\|_{\boldsymbol{V}_{1}} \leq C\|\nabla \times \boldsymbol{v}\|=C\|\nabla \times \boldsymbol{w}\| .
$$

We combine these two propositions to obtain the following lemma.

Lemma 3.1. Let $\boldsymbol{u}$ be in $\boldsymbol{L}^{2}(\Omega)$. Then there exists a decomposition

$$
\boldsymbol{u}=\nabla \times \boldsymbol{w}+\mu \nabla \psi
$$

where $\boldsymbol{w} \in \boldsymbol{V}_{1}$ and $\psi \in H^{1}(\Omega) / \mathbb{R}$ (the functions in $H_{1}$ with zero mean value). Furthermore

$$
\|\boldsymbol{w}\|_{\boldsymbol{V}_{1}} \leq C\|\nabla \times \boldsymbol{w}\|
$$

The above lemma will be used in the treatment of Problem 1. We will need the following for Problem 2.

Proposition 3.3. Let $\boldsymbol{u}$ be in $\boldsymbol{L}^{2}(\Omega)$. Then there exists a decomposition

$$
\boldsymbol{u}=\boldsymbol{v}+\mu \nabla \theta
$$

where $\boldsymbol{v} \in \boldsymbol{H}(\operatorname{div})$ satisfies $\nabla \cdot \boldsymbol{v}=0,(\boldsymbol{v} \cdot \boldsymbol{n}, 1)_{\Gamma_{i}}=0, i=0,1, \ldots, p$ and $\theta \in H_{2}$.

Proof. Let $\boldsymbol{u}$ be in $\boldsymbol{L}^{2}(\Omega)$ and let $\eta$ be the unique function in $H_{0}^{1}(\Omega)$ satisfying

$$
(\mu \nabla \eta, \nabla \phi)=(\boldsymbol{u}, \nabla \phi) \quad \text { for all } \phi \in H_{0}^{1}(\Omega) .
$$

We then set $\boldsymbol{v}=\boldsymbol{u}-\mu \nabla \eta-\mu \nabla \psi \equiv \boldsymbol{u}-\mu \nabla \theta$, with $\psi \in W_{d}$ to be determined. Clearly, $\boldsymbol{v}$ and $\boldsymbol{u}-\mu \nabla \eta$ are in $\boldsymbol{H}$ (div) and $\nabla \cdot \boldsymbol{v}=0$. Let $\left\{c_{i}\right\}$ be the solution of the nonsingular system $\sum_{j=1}^{p} c_{j}\left(\mu \nabla \psi_{i}, \nabla \psi_{j}\right)=((\boldsymbol{u}-\mu \nabla \eta) \cdot \boldsymbol{n}, 1)_{\Gamma_{i}}$, for $i=$ $1,2, \ldots, p$ and set $\psi=\sum_{i=1}^{p} c_{i} \psi_{i}$. Note that, by construction, $(\mu \nabla \psi \cdot \boldsymbol{n}, 1)_{\Gamma_{i}}=$ $((\boldsymbol{u}-\mu \nabla \eta) \cdot \boldsymbol{n}, 1)_{\Gamma_{i}}$ so $(\boldsymbol{v} \cdot \boldsymbol{n}, 1)_{\Gamma_{i}}=0$ for $i=0, \ldots, p$. This completes the proof of the proposition.

Lemma 3.2. Let $\boldsymbol{u}$ be in $\boldsymbol{L}^{2}(\Omega)$. Then there exists a decomposition

$$
\boldsymbol{u}=\nabla \times \boldsymbol{w}+\mu \nabla \theta
$$

where $\boldsymbol{w} \in \boldsymbol{V}_{2}$ and $\theta \in H_{2}$ and $(\nabla \times \boldsymbol{w} \cdot \boldsymbol{n}, 1)_{\Gamma_{i}}=0, i=1,2, \ldots, p$, Furthermore,

$$
\|\boldsymbol{w}\|_{\boldsymbol{V}_{2}} \leq C\|\nabla \times \boldsymbol{w}\| .
$$

Proof. Applying Proposition 3.3 since $\nabla \cdot \boldsymbol{v}=0$ and $(\boldsymbol{v} \cdot \boldsymbol{n}, 1)_{\Gamma_{i}}=0, i=1,2, \ldots, p$, we may use Theorem 3.4 of [15] to show that $\boldsymbol{v}=\nabla \times \boldsymbol{w}$ with $\boldsymbol{w}$ satisfying the above stated conditions. This completes the proof of the lemma. 


\section{A variational formulation of Problem 1}

In this section, we set up variational formulations of Problem 1 based in $\boldsymbol{L}^{2}(\Omega)$. We consider the more general problem

$$
\begin{aligned}
\nabla \times \mu^{-1} \boldsymbol{b} & =\boldsymbol{j} \text { in } \Omega, \\
\nabla \cdot \boldsymbol{b} & =g \text { in } \Omega, \\
\boldsymbol{b} \cdot \boldsymbol{n} & =\sigma \text { on } \Gamma .
\end{aligned}
$$

We can get a variational formulation of (4.1) by integration by parts. Since $C_{0}^{\infty}(\Omega)$ is dense in $\boldsymbol{V}_{1}$, the weak form of the first equation in 4.1] is

$$
\left(\mu^{-1} \boldsymbol{b}, \nabla \times \boldsymbol{w}\right)=\langle\boldsymbol{j}, \boldsymbol{w}\rangle, \quad \text { for all } \boldsymbol{w} \in \boldsymbol{V}_{1} .
$$

If $\boldsymbol{b} \in \boldsymbol{L}^{2}(\Omega)$ and $g \in L^{2}(\Omega)$, then $\boldsymbol{b}$ is in $\boldsymbol{H}$ (div) and we have the integration by parts formula

$$
\begin{aligned}
(\boldsymbol{b}, \nabla \phi) & =(\boldsymbol{b} \cdot \boldsymbol{n}, \phi)_{\Gamma}-(\nabla \cdot \boldsymbol{b}, \phi) \\
& =(\sigma, \phi)_{\Gamma}-(g, \phi) \equiv\langle l, \phi\rangle \quad \text { for all } \phi \in H_{1} .
\end{aligned}
$$

Here $l$ is the unique functional in $H_{1}^{*}$ defined by (4.2) (here we have implicitly assumed that $\left.\sigma \in H^{-1 / 2}(\Gamma)\right)$. We consider the weak formulation of (4.1) given by: Find $\boldsymbol{b} \in \boldsymbol{X}_{1} \equiv \boldsymbol{L}^{2}(\Omega)$ satisfying

$$
b_{1}(\boldsymbol{b} ;(\boldsymbol{w}, \phi)) \equiv\left(\mu^{-1} \boldsymbol{b}, \nabla \times \boldsymbol{w}\right)+(\boldsymbol{b}, \nabla \phi)=\langle\boldsymbol{j}, \boldsymbol{w}\rangle+\langle l, \phi\rangle,
$$

for all $(\boldsymbol{w}, \phi) \in \boldsymbol{V}_{1} \times H_{1} \equiv \boldsymbol{Y}_{1}$. This is our variational formulation of Problem 1 on $\boldsymbol{X}_{1} \times \boldsymbol{Y}_{1}$.

For vector or scalar functions $u, v$, let

$$
(v, w)_{\mu}=(\mu v, w)
$$

with the corresponding norm denoted by $\|\cdot\|_{\mu}$. We shall use similar notation with $\mu^{-1}$.

One can analyze the above problem by verifying the hypothesis of Theorem 2.1 It is obvious that $b_{1}(\cdot ; \cdot)$ is bounded and the inf-sup condition follows quite easily from Lemma 3.1 Indeed, for $\boldsymbol{u} \in \boldsymbol{L}^{2}(\Omega)$, let $\boldsymbol{u}=\boldsymbol{w}+\mu \nabla \psi$ be the decomposition. Since, $\boldsymbol{w} \in \boldsymbol{V}_{1}$ and $\psi \in H^{1}(\Omega),(\nabla \times \boldsymbol{w}, \mu \nabla \psi)_{\mu^{-1}}=0$. Hence,

$$
\|\boldsymbol{u}\|_{\mu^{-1}}^{2}=\|\nabla \times \boldsymbol{w}\|_{\mu^{-1}}^{2}+\|\nabla \psi\|_{\mu}^{2}=\frac{\left(\mu^{-1} \boldsymbol{u}, \nabla \times \boldsymbol{w}\right)^{2}}{\|\nabla \times \boldsymbol{w}\|_{\mu^{-1}}^{2}}+\frac{(\boldsymbol{u}, \nabla \psi)^{2}}{\|\nabla \psi\|_{\mu}^{2}} .
$$

Using the inequality in Lemma 3.1, we conclude that

$$
\|\boldsymbol{u}\|_{\mu^{-1}}^{2} \leq C \mu\left(\sup _{\boldsymbol{w} \in \boldsymbol{V}_{1}} \frac{\left(\mu^{-1} \boldsymbol{u}, \nabla \times \boldsymbol{w}\right)^{2}}{\|\boldsymbol{w}\|_{\boldsymbol{V}_{1}}^{2}}+\sup _{\psi \in H_{1} / \mathbb{R}} \frac{(\boldsymbol{u}, \nabla \psi)^{2}}{\|\nabla \psi\|_{\mu}^{2}}\right) .
$$

It follows from (3.2) that

$$
\|\boldsymbol{u}\|_{\mu^{-1}} \leq C \mu^{1 / 2} \sup _{(\boldsymbol{w}, \psi) \in \boldsymbol{Y}_{1}} \frac{b_{1}(\boldsymbol{u} ;(\boldsymbol{w}, \psi))}{\|(\boldsymbol{w}, \psi)\|_{\boldsymbol{Y}_{1}}}
$$

where

$$
\|(\boldsymbol{w}, \psi)\|_{\boldsymbol{Y}_{1}}^{2}=\left(\|\boldsymbol{w}\|_{\boldsymbol{V}_{1}}^{2}+\|\psi\|_{H_{1}}^{2}\right)^{1 / 2}
$$

Moreover, it is easy to see that $b_{1}(\boldsymbol{u} ;(\boldsymbol{w}, \phi))=0$ for all $\boldsymbol{u} \in \boldsymbol{L}^{2}(\Omega)$ if $\phi$ is constant and $\boldsymbol{w} \in \boldsymbol{V}_{1,0}$ where

$$
\boldsymbol{V}_{1,0}=\left\{\boldsymbol{w} \in \boldsymbol{V}_{1}: \boldsymbol{w}=\nabla \psi\right\}
$$


Thus, by the Theorem [2.1, (4.3) is uniquely solvable if

$$
\boldsymbol{j} \in \widetilde{\boldsymbol{V}}_{\mathbf{1}}^{*} \equiv\left\{\boldsymbol{j} \in \boldsymbol{V}_{1}^{*}:\langle\boldsymbol{j}, \boldsymbol{w}\rangle=0, \quad \text { for all } \boldsymbol{w} \in \boldsymbol{V}_{1,0}\right\}
$$

and

$$
l \in{\widetilde{H_{1}}}^{*} \equiv\left\{l \in H_{1}^{*}:\langle l, 1\rangle=0\right\} .
$$

For $\boldsymbol{v} \in \boldsymbol{L}^{2}(\Omega)$, we define $\operatorname{curl}_{\mu^{-1}} \boldsymbol{v} \in \boldsymbol{V}_{1}^{*}$ and $\operatorname{div}_{1} \boldsymbol{v} \in H_{1}^{*}$ by

$$
\left\langle\operatorname{curl}_{\mu^{-1}} \boldsymbol{v}, \boldsymbol{w}\right\rangle=\left(\mu^{-1} \boldsymbol{v}, \nabla \times \boldsymbol{w}\right) \quad \text { for all } \boldsymbol{w} \in \boldsymbol{V}_{1}
$$

and

$$
\left\langle\operatorname{div}_{1} \boldsymbol{v}, \phi\right\rangle=(\boldsymbol{v}, \nabla \phi) \quad \text { for all } \phi \in H_{1} .
$$

We can then rewrite (4.3) in the form: Find $\boldsymbol{b} \in \boldsymbol{L}^{2}(\Omega)$ satisfying

$$
\begin{aligned}
\operatorname{curl}_{\mu^{-1}} \boldsymbol{b} & =\boldsymbol{j}, \text { in } \boldsymbol{V}_{1}^{*}, \\
\operatorname{div}_{1} \boldsymbol{b} & =l, \text { in } H_{1}^{*} .
\end{aligned}
$$

For functionals $l \in{\widetilde{H_{1}}}^{*}$, we have

$$
\|l\|_{H_{1}^{*}}=\sup _{\phi \in H_{1} / \mathbb{R}} \frac{\langle l, \phi\rangle}{\|\nabla \phi\|_{\mu}} .
$$

We can restate the above in terms of the following theorem.

Theorem 4.1. The map $\boldsymbol{u} \rightarrow\left(\operatorname{curl}_{\mu^{-1}} \boldsymbol{u}, \operatorname{div}_{1} \boldsymbol{u}\right)$ is an isomorphism of $\boldsymbol{L}^{2}(\Omega)$ onto ${\widetilde{V_{1}}}^{*} \times{\widetilde{H_{1}}}^{*}$. Furthermore there are constants $C_{0}$ and $C_{1}$ not depending on $\mu$ satisfying

$$
C_{0} \mu_{1}^{-1}\|\boldsymbol{u}\|_{\mu^{-1}}^{2} \leq\left\|\operatorname{curl}_{\mu^{-1}} \boldsymbol{u}\right\|_{\boldsymbol{V}_{1}^{*}}^{2}+\left\|\operatorname{div}_{1} \boldsymbol{u}\right\|_{H_{1}^{*}}^{2} \leq C_{1} \mu_{0}^{-1}\|\boldsymbol{u}\|_{\mu^{-1}}^{2},
$$

for all $\boldsymbol{u} \in \boldsymbol{L}^{2}(\Omega)$.

Proof. From the above discussion and the definition of $\operatorname{curl}_{\mu^{-1}}$ and $\operatorname{div}_{1}$, it is immediate that the map $\boldsymbol{u} \rightarrow\left(\operatorname{curl}_{\mu^{-1}} \boldsymbol{u}, \operatorname{div}_{1} \boldsymbol{u}\right)$ maps $\boldsymbol{L}^{2}(\Omega)$ continuously into ${\widetilde{V_{1}}}^{*} \times{\widetilde{H_{1}}}^{*}$. The second inequality of (4.7) follows easily. The other inequality is just (4.4).

As in Section 2, we can define the dual based least-squares problem. We define the symmetric quadratic form

$$
\boldsymbol{A}_{1}(\boldsymbol{u}, \boldsymbol{v})=\left(\operatorname{curl}_{\mu^{-1}} \boldsymbol{u}, \operatorname{curl}_{\mu^{-1}} \boldsymbol{v}\right)_{\boldsymbol{V}_{1}^{*}}+\left(\operatorname{div}_{1} \boldsymbol{u}, \operatorname{div}_{1} \boldsymbol{v}\right)_{H_{1}^{*}}
$$

for all $\boldsymbol{u}, \boldsymbol{v} \in \boldsymbol{L}^{2}(\Omega)$ and the functional

$$
\langle\boldsymbol{l}, \boldsymbol{\phi}\rangle=\left(\boldsymbol{j}, \operatorname{curl}_{\mu^{-1}} \boldsymbol{\phi}\right)_{\boldsymbol{V}_{1}^{*}}+\left(l, \operatorname{div}_{1} \boldsymbol{\phi}\right)_{H_{1}^{*}} .
$$

From Theorem 2.2, the solution $\boldsymbol{b}$ of

$$
\boldsymbol{A}_{1}(\boldsymbol{b}, \boldsymbol{v})=\langle\boldsymbol{l}, \boldsymbol{v}\rangle \quad \text { for all } \boldsymbol{v} \in \boldsymbol{L}^{2}(\Omega) .
$$

coincides with that of 4.3) when $\boldsymbol{j} \in \widetilde{\boldsymbol{V}}_{1}^{*}$ and $l \in \widetilde{H}_{1}^{*}$.

It is possible to consider an alternative formulation where one solves for $\boldsymbol{h}$. In this case, one uses the two operators $\operatorname{curl}_{1}: \boldsymbol{L}^{2}(\Omega) \rightarrow \boldsymbol{V}_{1}^{*}$ and $\operatorname{div}_{\mu}: \boldsymbol{L}^{2}(\Omega) \rightarrow H_{1}^{*}$ defined by

$$
\begin{aligned}
\left\langle\operatorname{curl}_{1} \boldsymbol{v}, \boldsymbol{w}\right\rangle & =(\boldsymbol{v}, \nabla \times \boldsymbol{w}) \quad \text { for all } \boldsymbol{v} \in \boldsymbol{L}^{2}(\Omega), \boldsymbol{w} \in \boldsymbol{V}_{1}, \\
\left\langle\operatorname{div}_{\mu} \boldsymbol{v}, w\right\rangle & =(\mu \boldsymbol{v}, \nabla w) \quad \text { for all } \boldsymbol{v} \in \boldsymbol{L}^{2}(\Omega), w \in H_{1} .
\end{aligned}
$$

We note that operator $\operatorname{curl}_{1}$ defined above coincides with the distributional curl. 
The alternative version of Problem 1 is then: Find $\boldsymbol{h} \in \boldsymbol{L}^{2}(\Omega)$ satisfying

$$
\begin{aligned}
\operatorname{curl}_{1} \boldsymbol{h} & =\boldsymbol{j}, \text { in } \boldsymbol{V}_{1}^{*}, \\
\operatorname{div}_{\mu} \boldsymbol{h} & =\boldsymbol{l}, \text { in } H_{1}^{*} .
\end{aligned}
$$

The following result follows immediately from Theorem 4.1 by replacing $\boldsymbol{u}$ by $\mu \boldsymbol{u}$. The map $\boldsymbol{u} \rightarrow\left(\operatorname{curl}_{1} \boldsymbol{u}, \operatorname{div}_{\mu} \boldsymbol{u}\right)$ is an isomorphism of $\boldsymbol{L}^{2}(\Omega)$ onto $\widetilde{\boldsymbol{V}}_{\mathbf{1}}{ }^{*} \times{\widetilde{H_{1}}}^{*}$. Furthermore, by (4.7),

$$
C_{0} \mu_{1}^{-1}\|\boldsymbol{u}\|_{\mu}^{2} \leq \widetilde{\boldsymbol{A}}_{1}(\boldsymbol{u}, \boldsymbol{u}) \leq C_{1} \mu_{0}^{-1}\|\boldsymbol{u}\|_{\mu}^{2} \quad \text { for all } \boldsymbol{u} \in \boldsymbol{L}^{2}(\Omega) .
$$

Here $\widetilde{\boldsymbol{A}}_{1}(\cdot, \cdot)$ is defined by

$$
\widetilde{\boldsymbol{A}}_{1}(\boldsymbol{u}, \boldsymbol{w})=\left(\operatorname{curl}_{1} \boldsymbol{u}, \operatorname{curl}_{1} \boldsymbol{w}\right)_{\boldsymbol{V}_{1}^{*}}+\left(\operatorname{div}_{\mu} \boldsymbol{u}, \operatorname{div}_{\mu} \boldsymbol{w}\right)_{H_{1}^{*}} .
$$

Remark 4.1. It is possible to take $H_{1}=H^{1}(\Omega) / \mathbb{R}$. In this case, one loses the compatibility condition $\langle l, 1\rangle=0$. In addition, one has to impose the mean value condition on the subsequent approximation spaces complicating the discretization process.

\section{A variational formulation of Problem 2}

In this section, we consider a div-curl system with the second boundary condition. Specifically, we consider the following more general problem

$$
\begin{aligned}
\nabla \times \boldsymbol{h} & =\boldsymbol{j} \text { in } \Omega, \\
\nabla \cdot \mu \boldsymbol{h} & =g \text { in } \Omega, \\
\boldsymbol{h} \times \boldsymbol{n} & =\boldsymbol{\sigma} \text { on } \Gamma \\
(\mu \boldsymbol{h} \cdot \boldsymbol{n}, 1)_{\Gamma_{i}} & =C_{i}, i=1,2, \ldots, p .
\end{aligned}
$$

We will develop a weak formulation of (5.1) where $\boldsymbol{h}$ is in $\boldsymbol{L}^{2}(\Omega)$ and $\boldsymbol{j}, g$, and $\boldsymbol{\sigma}$ are functionals on appropriate spaces (discussed below). We pose the problem in the variational framework of Section 2. We use $\boldsymbol{X}_{2} \equiv \boldsymbol{L}^{2}(\Omega)$ and $\boldsymbol{Y}_{2} \equiv \boldsymbol{V}_{2} \times H_{2}$. We define the bilinear form $b$ by integration by parts. The weak form of the first equation in 5.1 is

$$
(\boldsymbol{h}, \nabla \times \boldsymbol{w})=\langle\boldsymbol{j}, \boldsymbol{w}\rangle-\left\langle\boldsymbol{\sigma},\left.\boldsymbol{w}\right|_{\Gamma}\right\rangle \equiv\langle\boldsymbol{J}, \boldsymbol{w}\rangle, \quad \text { for all } \boldsymbol{w} \in \boldsymbol{V}_{2} .
$$

For $\boldsymbol{J}$ to be in $\boldsymbol{V}_{2}^{*}$, we need $\boldsymbol{j} \in \boldsymbol{V}_{2}^{*}$ and $\boldsymbol{\sigma} \in \boldsymbol{H}^{-1 / 2}(\Gamma)$, the dual of $\boldsymbol{H}^{1 / 2}(\Gamma)=$ $\left\{\left.\phi\right|_{\Gamma}: \phi \in V_{2}\right\}$. A weak form of the second equation in (5.1) is

$$
(\mu \boldsymbol{h}, \nabla \phi)=(\mu \boldsymbol{h} \cdot \boldsymbol{n}, \phi)_{\Gamma}-\langle g, \phi\rangle, \quad \text { for all } \phi \in H_{2} .
$$

If $\phi=\phi_{0}+\sum_{i=1}^{p} \alpha_{i} \psi_{i}$ with $\phi_{0} \in H_{0}^{1}(\Omega)$, then

$$
(\mu \boldsymbol{h} \cdot \boldsymbol{n}, \phi)_{\Gamma}=\sum_{i=1}^{p} C_{i} \alpha_{i} .
$$

Thus,

$$
(\mu \boldsymbol{h}, \nabla \phi)=-\langle g, \phi\rangle+\sum_{i=1}^{p} C_{i} \alpha_{i} \equiv\langle l, \phi\rangle, \quad \text { for all } \phi \in H_{2} .
$$

Clearly, $l \in H_{2}^{*}$ provided that $g \in H_{2}^{*}$.

In view of the above, we consider the weak formulation of (5.1) given by: Find $\boldsymbol{h} \in \boldsymbol{X}_{2}$ satisfying

$$
b_{2}(\boldsymbol{h} ;(\boldsymbol{w}, \phi)) \equiv(\boldsymbol{h}, \nabla \times \boldsymbol{w})+(\mu \boldsymbol{h}, \nabla \phi)=\langle\boldsymbol{J}, \boldsymbol{w}\rangle+\langle l, \phi\rangle,
$$


for all $(\boldsymbol{w}, \phi) \in \boldsymbol{Y}_{2} \equiv \boldsymbol{V}_{2} \times H_{2}$. This is our variational formulation of Problem 2 on $\boldsymbol{X}_{2} \times \boldsymbol{Y}_{2}$.

One can analyze the weak formulation (5.2) above by verifying the hypothesis of Theorem 2.1. It is obvious that $b_{2}(\cdot ; \cdot)$ is bounded and the inf-sup condition follows quite easily from Lemma 3.2. In fact, using the decomposition of Lemma 3.2 applied to $\mu \boldsymbol{u} \in \boldsymbol{L}^{2}(\Omega)$, we have $\left(\mu^{-1} \nabla \times \boldsymbol{w}, \nabla \psi\right)_{\mu}=0$. Thus

$$
\|\boldsymbol{u}\|_{\mu}^{2}=\|\nabla \times \boldsymbol{w}\|_{\mu^{-1}}^{2}+\|\nabla \psi\|_{\mu}^{2}=\frac{(\boldsymbol{u}, \nabla \times \boldsymbol{w})^{2}}{\|\nabla \times \boldsymbol{w}\|_{\mu^{-1}}^{2}}+\frac{(\mu \boldsymbol{u}, \nabla \psi)^{2}}{\|\nabla \psi\|_{\mu}^{2}} .
$$

Using the inequality in Lemma 3.2, we conclude that

$$
\|\boldsymbol{u}\|_{\mu}^{2} \leq C \mu_{1}\left(\sup _{\boldsymbol{w} \in \boldsymbol{V}_{2}} \frac{(\boldsymbol{u}, \nabla \times \boldsymbol{w})^{2}}{\|\boldsymbol{w}\|_{\boldsymbol{V}_{2}}^{2}}+\sup _{\psi \in H_{2}} \frac{(\mu \boldsymbol{u}, \nabla \psi)^{2}}{\|\nabla \psi\|_{\mu}^{2}}\right),
$$

from which it follows that

$$
\|\boldsymbol{u}\|_{\mu} \leq C \mu_{1}^{1 / 2} \sup _{(\boldsymbol{w}, \psi) \in \boldsymbol{Y}_{2}} \frac{b_{2}(\boldsymbol{u} ;(\boldsymbol{w}, \psi))}{\|(\boldsymbol{w}, \psi)\|_{\boldsymbol{Y}_{2}}}
$$

where

$$
\|(\boldsymbol{w}, \psi)\|_{\boldsymbol{Y}_{2}}^{2}=\left(\|\boldsymbol{w}\|_{\boldsymbol{V}_{2}}^{2}+\|\nabla \psi\|_{\mu}^{2}\right)^{1 / 2}
$$

Moreover, it is easy to see that $b_{2}(\boldsymbol{u} ;(\boldsymbol{w}, \phi))=0$ for all $\boldsymbol{u} \in \boldsymbol{L}^{2}(\Omega)$ if $\phi=0$ and $\boldsymbol{w} \in \boldsymbol{V}_{2,0}$ where

$$
\boldsymbol{V}_{2,0}=\left\{\boldsymbol{w} \in \boldsymbol{V}_{2}: \boldsymbol{w}=\nabla \psi\right\} .
$$

Thus, by the Theorem [2.1, (5.2) is uniquely solvable if $\boldsymbol{J} \in \widetilde{\boldsymbol{V}}_{\mathbf{2}}^{*} \equiv\left\{\boldsymbol{J} \in \boldsymbol{V}_{2}^{*}\right.$ : $\langle\boldsymbol{J}, \boldsymbol{w}\rangle=0, \quad$ for all $\left.\boldsymbol{w} \in \boldsymbol{V}_{2,0}\right\}$ and $l \in H_{2}^{*}$.

For $\boldsymbol{v} \in \boldsymbol{L}^{2}(\Omega)$, we define $\operatorname{curl}_{1} \boldsymbol{v} \in \boldsymbol{V}_{2}^{*}$ and $\operatorname{div}_{\mu} \boldsymbol{v} \in H_{2}^{*}$ by

$$
\left\langle\operatorname{curl}_{1} \boldsymbol{v}, \boldsymbol{w}\right\rangle=(\boldsymbol{v}, \nabla \times \boldsymbol{w}) \quad \text { for all } \boldsymbol{w} \in \boldsymbol{V}_{2}
$$

and

$$
\left\langle\operatorname{div}_{\mu} \boldsymbol{v}, \phi\right\rangle=(\mu \boldsymbol{v}, \nabla \phi) \quad \text { for all } \phi \in H_{2} .
$$

We can then rewrite (5.2) in the form: Find $\boldsymbol{h} \in \boldsymbol{L}^{2}(\Omega)$ satisfying

$$
\begin{aligned}
\operatorname{curl}_{1} \boldsymbol{h} & =\boldsymbol{J}, \text { in } \boldsymbol{V}_{2}^{*}, \\
\operatorname{div}_{\mu} \boldsymbol{h} & =l, \text { in } H_{2}^{*} .
\end{aligned}
$$

For functionals $l \in H_{2}^{*}$, we will use the equivalent norm

$$
\|l\|_{H_{2}^{*}}=\sup _{\phi \in H_{2}} \frac{\langle l, \phi\rangle}{\|\nabla \phi\|_{\mu}} .
$$

Using the definitions of $\boldsymbol{V}_{2}$ and $H_{2}$ for Problem 2, we can restate the above in terms of the following theorem.

Theorem 5.1. The map $\boldsymbol{u} \rightarrow\left(\operatorname{curl}_{1} \boldsymbol{u}, \operatorname{div}_{\mu} \boldsymbol{u}\right)$ is an isomorphism of $\boldsymbol{L}^{2}(\Omega)$ onto ${\widetilde{V_{2}}}^{*} \times H_{2}^{*}$. Furthermore there are constants $C_{0}$ and $C_{1}$ not depending on $\mu$ satisfying

$$
C_{0} \mu_{1}^{-1}\|\boldsymbol{u}\|_{\mu}^{2} \leq\left\|\operatorname{curl}_{1} \boldsymbol{u}\right\|_{V_{2}^{*}}^{2}+\left\|\operatorname{div}_{\mu} \boldsymbol{u}\right\|_{H_{2}^{*}}^{2} \leq C_{1} \mu_{0}^{-1}\|\boldsymbol{u}\|_{\mu}^{2},
$$

for all $\boldsymbol{u} \in \boldsymbol{L}^{2}(\Omega)$.

Proof. From the above discussion and the definition of $\operatorname{curl}_{1}$ and $\operatorname{div}_{\mu}$, it is immediate that the map $\boldsymbol{u} \rightarrow\left(\operatorname{curl}_{1} \boldsymbol{u}, \operatorname{div}_{\mu} \boldsymbol{u}\right)$ maps $\boldsymbol{L}^{2}(\Omega)$ continuously into $\widetilde{\boldsymbol{V}}_{\mathbf{2}}^{*} \times H_{2}^{*}$. The second inequality of (5.5) follows easily. The other inequality is just (5.3). 
We denote by $(\cdot, \cdot)_{H_{2}^{*}}$ and $(\cdot, \cdot)_{\boldsymbol{V}_{2}^{*}}$ the inner products corresponding to $\|\cdot\|_{H_{2}^{*}}$ and $\|\cdot\|_{V_{2}^{*}}$, respectively. As in Section 2 we can define the dual based least-squares problem. We define the symmetric quadratic form

$$
\boldsymbol{A}_{2}(\boldsymbol{u}, \boldsymbol{v})=\left(\operatorname{curl}_{1} \boldsymbol{u}, \operatorname{curl}_{1} \boldsymbol{v}\right)_{\boldsymbol{V}_{2}^{*}}+\left(\operatorname{div}_{\mu} \boldsymbol{u}, \operatorname{div}_{\mu} \boldsymbol{v}\right)_{H_{2}^{*}}
$$

for all $\boldsymbol{u}, \boldsymbol{v} \in \boldsymbol{L}^{2}(\Omega)$ and the functional

$$
\langle\boldsymbol{l}, \boldsymbol{\phi}\rangle=\left(\boldsymbol{J}, \operatorname{curl}_{1} \phi\right)_{\boldsymbol{V}_{2}^{*}}+\left(l, \operatorname{div}_{\mu} \phi\right)_{H_{2}^{*}} .
$$

From Theorem 2.2, the solution $\boldsymbol{b}$ of

$$
\boldsymbol{A}_{2}(\boldsymbol{b}, \boldsymbol{v})=\langle\boldsymbol{l}, \boldsymbol{v}\rangle \quad \text { for all } \boldsymbol{v} \in \boldsymbol{L}^{2}(\Omega)
$$

coincides with that of (5.2) when the $\boldsymbol{J} \in \widetilde{\boldsymbol{V}}_{2}^{*}$.

Remark 5.1. We note that Lemma 3.2 holds for reasonable nonsimply connected domains and hence all of the analysis in this section applies in that case. In contrast, Proposition 3.1 does not and the method of Section 4 must be modified. To do this, one introduces cuts $\left\{\Sigma_{j}\right\}, j=1, \ldots, J$, into the domain so that the resulting domain $\Omega_{0}$ is simply connected (see, e.g. [1]). Analogous to the space $W_{d}$, we define a finite dimensional space $W_{n}$ spanned by the functions $\left\{\zeta_{j}\right\}$ satisfying

$$
\begin{gathered}
-\nabla \cdot \mu \nabla \zeta_{j}=0 \text { on } \Omega_{0}, \\
\frac{\partial \zeta_{j}}{\partial \boldsymbol{n}}=0 \text { on } \partial \Omega \\
{\left[\zeta_{j}\right]=\delta_{i j} \text { and }\left[\mu \frac{\partial \zeta_{j}}{\partial \boldsymbol{n}}\right]=0 \text { on } \Sigma_{i} .}
\end{gathered}
$$

Here [·] denotes the jump across the cut. Let $\widetilde{\nabla} \zeta_{j}$ denote the distributional gradient of $\zeta_{j}$ with respect to $\Omega_{0}$. Then $\mu \widetilde{\nabla} \zeta$ satisfies (4.1) with homogeneous right-hand side 1 . Thus for unique solvability, one needs to add additional equations, for example,

$$
(\boldsymbol{b} \cdot \boldsymbol{n}, 1)_{\Sigma_{j}}=C_{j}, \quad j=1, \ldots, J .
$$

In this case, we take $H_{1}=H^{1}(\Omega) \oplus W_{n}$ and everything goes through.

\section{The CONSTRUCTION OF STABLE APPROXIMATION PAIRS}

In this section, we show one way of constructing stable approximation pairs for the variational Problems 1 and 2. Specifically, we will define approximation pairs $\left(\boldsymbol{X}_{h}, \boldsymbol{Y}_{h}\right)$ satisfying the discrete inf-sup condition (2.6).

For convenience, we restrict our discussion to tetrahedral partitioning of polyhedral domains $\Omega \subset \mathbb{R}^{3}$ and assume that $\mu$ is piecewise constant. The constructions extends to other element shapes as well as div-curl problems on domains in $\mathbb{R}^{2}$.

We start with an admissible mesh of tetrahedra, $\Omega=\bigcup \tau_{i}$ (for convenience, we shall always consider tetrahedra to be closed) which align with the jumps in $\mu$ so that $\mu$ is constant on the interior of each tetrahedron. We shall assume that the "triangulation" is locally quasi-uniform. By this we mean that there is a constant $c_{0}$ not depending on $h$ (the diameter of the largest element in $\mathbb{T} \equiv\left\{\tau_{i}\right\}$ ) such that

$$
c_{0} h_{\tau}^{0} \geq h_{\tau}, \quad \text { for all } \tau \in \mathbb{T} .
$$

Here $h_{\tau}$ denotes the diameter of $\tau$ and $h_{\tau}^{0}$ denotes the diameter of the largest ball which can be inscribed in $\tau$. 
For all of the formulations in the preceding two sections, we require approximation subspaces for $\boldsymbol{L}^{2}(\Omega)$. For each component, we will use piecewise linear or constant functions with respect to the mesh $\mathbb{T}$. Extensions to higher order finite element approximations are possible. We allow the order of the polynomials to change from element to element. The resulting approximation spaces will be, in general, discontinuous. For example, to avoid Gibbs' phenomenon, we might employ discontinuous functions at the interfaces (where $\mu$ is discontinuous). In addition, we use discontinuous spaces at the faces between tetrahedra with different polynomial order. However, in regions where the solution is smooth, we suggest the use of continuous functions of fixed order. The order of approximation can be different for each component on each element. We denote by $\boldsymbol{X}_{h}$ the resulting discrete space.

We first illustrate the constructions for the lowest order approximation where $\boldsymbol{X}_{h}$ consists of piecewise constant vector functions. We need to construct the test spaces $\boldsymbol{Y}_{h}=\boldsymbol{V}_{h} \times H_{h}$. The idea is to start with $C^{0}$-piecewise linear finite element functions satisfying the appropriate boundary condition. We consider the case of Problem 1 when $H=H^{1}(\Omega)$ and define $H_{h}$. The definition of $\boldsymbol{V}_{h}$ will be considered later. We set $H_{h}^{0}$ to be the space of piecewise linear continuous functions with respect to our mesh $\mathbb{T}$. Subsequently, $H_{h}$ will be defined by enriching $H_{h}^{0}$ by face bubble functions.

Let $\mathbb{F}=\{F\}$ denote the collection of faces of the mesh $\mathbb{T}$. For $F \in \mathbb{F}$, we start with the nontrivial cubic polynomial $p$ defined on $F$ which vanishes on the boundary of $F$ and is one on the barycenter of $F$ (this is a constant times the product of the barycentric coordinate functions on the face, i.e., the functions which are linear, one on one vertex and zero on the remainder.). We extend $p$ to $\Omega$ by first setting $\tilde{p}=0$ on all tetrahedra not having $F$ as a face. Let $\tau$ be a tetrahedron having $F$ as a face and let $l_{i}(x), i=1,2,3,4$, denote the barycentric coordinate for $x \in \tau$ where $l_{4}$ corresponds to the vertex not on $F$. We then define $\tilde{p}$ on $\tau$ by

$$
\tilde{p}(x)=l_{1}(x) l_{2}(x) l_{3}(x)
$$

and we define $B_{F}$ to be the one dimensional space generated by $\tilde{p}(x)$. Clearly, $\tilde{p}$ vanishes on the boundary of the union of the two tetrahedra having $F$ as a face so that $\tilde{p}$ extended by zero is in $H^{1}(\Omega)$. If $F$ is a boundary face we use the same construction but in this case the support of $F$ is contained in the single tetrahedron which has $F$ as a face.

We define the enriched space $H_{h} \equiv H_{h}^{0} \oplus H_{h}^{\mathbb{F}}$ where $H_{h}^{\mathbb{F}}=\oplus_{F} B_{F}$. For $v \in H_{h}$, let $v=v_{0}+v_{\mathbb{F}}$ be the unique decomposition with $v_{0} \in H_{h}^{0}$ and $v_{\mathbb{F}} \in H_{h}^{\mathbb{F}}$. We further decompose $v_{\mathbb{F}}=\sum_{F} w_{F}$ with $w_{F} \in B_{F}$.

The following inequalities are obtained easily by mapping to the reference tetrahedron and applying equivalence of norms on finite dimensional spaces.

$$
\|v\|_{H^{1}(\Omega)}^{2} \approx\left\|v_{0}\right\|_{H^{1}(\Omega)}^{2}+\left\|v_{\mathbb{F}}\right\|_{H^{1}(\Omega)}^{2}, \quad \text { for all } v \in H_{h},
$$

and

$$
\left\|v_{\mathbb{F}}\right\|_{H^{1}(\Omega)}^{2} \approx \sum_{F}\left\|w_{F}\right\|_{H^{1}(\Omega)}^{2} .
$$

In the above inequalities, we use $a \approx b$ to mean that there are positive constants $c_{0}$ and $c_{1}$ not depending on $h$ satisfying $c_{0} a \leq b \leq c_{1} a$. We then have the following lemma. 
Lemma 6.1. Let $H=H^{1}(\Omega)$ and $\boldsymbol{X}_{h}$ and let $H_{h}$ be defined as above. Then there is a constant $C$ not depending on $h$ satisfying

$$
\sup _{\phi \in H} \frac{(\boldsymbol{u}, \nabla \phi)}{\|\phi\|_{H}} \leq C \sup _{\phi \in H_{h}} \frac{(\boldsymbol{u}, \nabla \phi)}{\|\phi\|_{H}}, \quad \text { for all } \boldsymbol{u} \in \boldsymbol{X}_{h} .
$$

Proof. Let $\boldsymbol{u}$ be in $X_{h}$ and let $\phi$ be in $H$. It suffices to construct $\phi_{h} \in H_{h}$ satisfying

$$
(\boldsymbol{u}, \nabla \phi)=\left(\boldsymbol{u}, \nabla \phi_{h}\right)
$$

and

$$
\left\|\phi_{h}\right\|_{H} \leq C\|\phi\|_{H} .
$$

It is well known that there exists a stable approximation operator (see, e.g., [9]) $I_{h}: L^{2}(\Omega) \rightarrow H_{h}^{0}$ satisfying

$$
\sum_{\tau \in \mathbb{T}}\left(h_{\tau}^{-2}\left\|I_{h} \phi-\phi\right\|_{L^{2}(\tau)}^{2}+\left\|\nabla\left(I_{h} \phi\right)\right\|_{L^{2}(\tau)}^{2}\right) \leq C\|\phi\|_{H^{1}(\Omega)}^{2} .
$$

We shall define

$$
\phi_{h}=I_{h} \phi+\phi_{\mathbb{F}}
$$

with appropriate choice for $\phi_{\mathbb{F}} \in H_{h}^{\mathbb{F}}$. Let $\psi=\phi-I_{h} \phi$ and set $w_{F} \in B_{F}$ by

$$
\left(1, w_{F}\right)_{F}=(1, \psi)_{F}
$$

where $(\cdot, \cdot)_{F}$ denotes the $L^{2}(F)$ inner product on $F$. This notation will also be used for other domains and for vector functions. The function $w_{F}$ is well defined since the basis function for $B_{F}(\tilde{p}$ defined in (6.1)) is positive almost everywhere on $F$.

Arguments involving mapping to a reference element imply that

$$
\left\|w_{F}\right\|_{L^{2}(F)} \leq C\|\psi\|_{L^{2}(F)} .
$$

We define $\phi_{\mathbb{F}}=\sum_{F} w_{F}$. We then have,

$$
\left\|\phi_{\mathbb{F}}\right\|_{H}^{2} \leq C \sum_{F}\left\|w_{F}\right\|_{H}^{2} \leq C \sum_{F} h_{\tau}^{-1}\left\|w_{F}\right\|_{L^{2}(F)}^{2}
$$

where $\tau$ is either of the tetrahedra having $F$ as a face. We then have

$$
\begin{aligned}
\left\|\phi_{\mathbb{F}}\right\|_{H}^{2} & \leq C \sum_{F} h_{\tau}^{-1}\|\psi\|_{L^{2}(F)}^{2} \\
& \leq C \sum_{\tau}\left(h_{\tau}^{-2}\|\psi\|_{L^{2}(\tau)}^{2}+\|\psi\|_{H^{1}(\tau)}^{2}\right) \leq C\|\phi\|_{H}^{2} .
\end{aligned}
$$

This shows that (6.4) holds for $\phi_{h}$ given by (6.6).

To show that (6.3) holds, it suffices to check that

$$
(\boldsymbol{u}, \nabla \psi)=\left(\boldsymbol{u}, \nabla \phi_{F}\right) \text {. }
$$

Integration by parts gives

$$
(\boldsymbol{u}, \nabla \psi)=\sum_{\tau}(\boldsymbol{u} \cdot \boldsymbol{n}, \psi)_{\partial \tau}
$$

Now, since $\boldsymbol{u} \cdot \boldsymbol{n}$ is constant on each face of $\partial \tau$, (6.7) implies

$$
(\boldsymbol{u} \cdot \boldsymbol{n}, \psi)_{\partial \tau}=\left(\boldsymbol{u} \cdot \boldsymbol{n}, \phi_{\mathbb{F}}\right)_{\partial \tau}
$$

from which (6.9) immediately follows. This completes the proof of the lemma. 
The construction and proof extend trivially to the case of $H=H_{0}^{1}(\Omega)$. We define $H_{h}^{0}$ to be continuous piecewise linear functions which vanish on $\partial \Omega$ and do not introduce any face bubble functions for those faces on $\partial \Omega$. Thus we have the following lemma.

Lemma 6.2. Let $H=H_{0}^{1}(\Omega)$ and let $\boldsymbol{X}_{h}$ and $H_{h}$ be defined as above. Then there is a constant $C$ not depending on $h$ satisfying

$$
\sup _{\phi \in H} \frac{(\mu \boldsymbol{u}, \nabla \phi)}{\|\phi\|_{H}} \leq C \sup _{\phi \in H_{h}} \frac{(\mu \boldsymbol{u}, \nabla \phi)}{\|\phi\|_{H}}, \quad \text { for all } \boldsymbol{u} \in \boldsymbol{X}_{h} .
$$

Remark 6.1. It is possible to deal with the case when $\mu$ is not constant. In this case, we need to add additional face and element bubble functions similar to those used in the constructions leading to Lemma 6.5 below.

The construction and proof also extend to pairs for $\boldsymbol{V}$. For $\boldsymbol{V}=\left(H_{0}^{1}(\Omega)^{3}\right)$ we can take three copies of the spaces previously constructed. The analysis proceeds by applying the constructions of the lemmas for each component. Indeed, for $\boldsymbol{\phi} \in \boldsymbol{V}$, we set $\boldsymbol{\psi}=\boldsymbol{\phi}-I_{h} \boldsymbol{\phi}$ and we define $\boldsymbol{\phi}_{\mathbb{F}}$ in $\left(H_{h}^{\mathbb{F}}\right)^{3}$ satisfying

$$
\begin{aligned}
\left(\mu^{-1} \boldsymbol{u}, \nabla \times \boldsymbol{\psi}\right) & =\sum_{\tau}\left(\mu^{-1} \boldsymbol{u} \times \boldsymbol{n}, \boldsymbol{\psi}\right)_{\partial \tau} \\
& =\sum_{\tau}\left(\mu^{-1} \boldsymbol{u} \times \boldsymbol{n}, \boldsymbol{\phi}_{\mathbb{F}}\right)_{\partial \tau} .
\end{aligned}
$$

We define the space $\boldsymbol{V}_{h} \equiv\left(H_{h}^{0}\right)^{3} \oplus\left(H_{h}^{\mathbb{F}}\right)^{3}$.

It is clear that one could instead use face bubble vector functions which have a zero normal component; however, adding face bubbles with nonzero normal components does not degrade the approximation and is simpler to implement. The case in which $\boldsymbol{V}=\left(H^{1}(\Omega)^{3}\right)$ and $\mu=1$ is similar. Thus we have the following lemmas.

Lemma 6.3. Let $\boldsymbol{V}=\left(H_{0}^{1}(\Omega)^{3}\right)$ and let $\boldsymbol{X}_{h}$ and $\boldsymbol{V}_{h}$ be defined as above. Then there is a constant $C$ not depending on $h$ satisfying

$$
\sup _{\boldsymbol{\phi} \in \boldsymbol{V}} \frac{\left(\mu^{-1} \boldsymbol{u}, \nabla \times \boldsymbol{\phi}\right)}{\|\boldsymbol{\phi}\|_{\boldsymbol{V}}} \leq C \sup _{\boldsymbol{\phi} \in \boldsymbol{V}_{h}} \frac{\left(\mu^{-1} \boldsymbol{u}, \nabla \times \boldsymbol{\phi}\right)}{\|\boldsymbol{\phi}\|_{\boldsymbol{V}}}, \quad \text { for all } \boldsymbol{u} \in \boldsymbol{X}_{h} .
$$

Lemma 6.4. Let $\boldsymbol{V}=\left(H^{1}(\Omega)^{3}\right)$ and let $\boldsymbol{X}_{h}$ and $\boldsymbol{V}_{h}$ be defined as above. Then there is a constant $C$ not depending on $h$ satisfying

$$
\sup _{\boldsymbol{\phi} \in \boldsymbol{V}} \frac{(\boldsymbol{u}, \nabla \times \boldsymbol{\phi})}{\|\boldsymbol{\phi}\|_{\boldsymbol{V}}} \leq C \sup _{\boldsymbol{\phi} \in \boldsymbol{V}_{h}} \frac{(\boldsymbol{u}, \nabla \times \boldsymbol{\phi})}{\|\boldsymbol{\phi}\|_{\boldsymbol{V}}}, \quad \text { for all } \boldsymbol{u} \in \boldsymbol{X}_{h} .
$$

We next consider the case when $\boldsymbol{X}_{h}$ consists of linear functions on the tetrahedra. As above, we first consider the case when $H=H^{1}(\Omega)$. If $\tau \in \mathbb{T}$, we set $B_{\tau}$ to be the one dimensional space spanned by the nontrivial fourth order polynomial defined on $\tau$ which vanishes on $\partial \tau$ (it is a multiple of the product of the barycentric coordinate functions associated with $\tau$ ). It is extended by zero outside of $\tau$. Then we define the space of element bubble functions by $H_{h}^{E}=\bigoplus B_{\tau}, \tau \in \mathbb{T}$.

We shall also need a larger set of face bubble functions. For the faces $F$ of elements in $\mathbb{T}$ we define the face space as follows. Let $b_{F}$ denote the set of functions defined on $\mathbb{R}^{3}$ which are linear on $F$ and constant along lines parallel to the normal of $F$. Let $p(x)$ be in $b_{F}$ and define $\tilde{p}$ by

- $\tilde{p}=0$ on all tetrahedra not having $F$ as a face. 
- If $\tau$ is a tetrahedron having $F$ as a face, $\tilde{p}(x)=l_{1}(x) l_{2}(x) l_{3}(x) p(x)$ on $\tau$ where $l_{i}$ are the barycentric coordinate functions on $\tau$ corresponding to the three vertices on $F$.

We note that $\tilde{p}(x)$ is continuous on $\Omega$. We set $B_{F}$ to be the span of such functions (over $p \in b_{F}$ ) and set $H_{h}^{\mathbb{F}}=\bigoplus_{F \in \mathbb{F}} B_{F}$. Clearly, the functions in $B_{F}$ are piecewise polynomials of degree four.

Lemma 6.5. Let $\boldsymbol{X}_{h}$ be the space consisting of vectors with piecewise linear components with respect to the triangulation $\mathbb{T}$. Let $H_{h}=H_{h}^{0} \oplus H_{h}^{\mathbb{F}} \oplus H_{h}^{E}$. Then (6.2) holds for the pair $\boldsymbol{X}_{h}, H_{h}$.

Proof. As is the case of Lemma6.1, given $\phi \in H$, we need only construct $\phi_{h} \in H_{h}$ satisfying (6.3) and (6.4). In this case, we set $\phi_{h}=I_{h} \phi+\phi_{\mathbb{F}}+\phi_{E}$ with $\phi_{\mathbb{F}} \in H_{h}^{\mathbb{F}}$ and $\phi_{E} \in H_{h}^{E}$.

We first define $\phi_{\mathbb{F}}=\sum_{F} w_{F}$ where $w_{F} \in B_{F}$ will be chosen below. For the faces $F$, we set $w_{F} \in B_{F}$ by

$$
\left(p, w_{F}\right)_{F}=(p, \psi)_{F}, \quad \text { for all linear } p,
$$

where $\psi=\phi-I_{h} \phi$. This problem is solvable since $B_{F}$ is obtained from the space of linear functions on $F$ by multiplication by the almost everywhere positive function $l_{1} l_{2} l_{3}$. Thus, (6.7) and (6.13) imply that for any $\boldsymbol{u} \in \boldsymbol{X}_{h}$,

$$
(\boldsymbol{u} \cdot \boldsymbol{n}, \phi)_{F}=\left(\boldsymbol{u} \cdot \boldsymbol{n}, I_{h} \phi+\phi_{\mathbb{F}}\right)_{F}=\left(\boldsymbol{u} \cdot \boldsymbol{n}, \phi_{h}\right)_{F} .
$$

The above equation is valid if the trace of $\boldsymbol{u}$ is taken from either tetrahedron having $F$ as a face.

Finally, we define $\phi_{E}$. Set $\theta=\phi-I_{h} \phi-\phi_{\mathbb{F}}$. For $\tau \in \mathbb{T}$, we define $w_{\tau} \in B_{\tau}$ by

$$
\left(1, w_{\tau}\right)_{\tau}=(1, \theta)_{\tau}
$$

and we set $\phi_{E}=\sum_{\tau \in \tilde{\mathbb{T}}} w_{\tau}$. Since, for any $\boldsymbol{u} \in \boldsymbol{X}_{h}, \nabla \cdot \boldsymbol{u}$ is a constant,

$$
(\nabla \cdot \boldsymbol{u}, \phi)=\left(\nabla \cdot \boldsymbol{u}, \phi_{h}\right)
$$

Combining the above inequalities and integration by parts show that (6.3) holds.

To complete the proof, we need only check that (6.4) holds. As in the lower order case, the following identities follow from mapping to the reference tetrahedron:

$$
\begin{gathered}
\left\|\phi_{\mathbb{F}}\right\|_{H^{1}(\Omega)}^{2} \approx \sum_{\tau} h_{\tau}^{-2}\left\|\phi_{\mathbb{F}}\right\|_{L^{2}(\tau)}^{2} \approx \sum_{F}\left\|w_{F}\right\|_{H^{1}(\Omega)}^{2} \approx \sum_{F} h_{\tau}^{-1}\left\|w_{F}\right\|_{L^{2}(F)}^{2}, \\
\left\|\phi_{E}\right\|_{H^{1}(\Omega)}^{2}=\sum_{\tau \in \widetilde{\mathbb{T}}}\left\|w_{\tau}\right\|_{H^{1}(\tau)}^{2} \approx \sum_{\tau \in \widetilde{\mathbb{T}}} h_{\tau}^{-2}\left\|w_{\tau}\right\|_{L^{2}(\tau)}^{2},
\end{gathered}
$$

and finally

$$
\left\|w_{F}\right\|_{L^{2}(F)} \leq C\|\psi\|_{L^{2}(F)} \text { and }\left\|w_{\tau}\right\|_{L^{2}(\tau)} \leq C\|\theta\|_{L^{2}(\tau)} .
$$

Exactly as in the proof of Lemma6.1, we get

$$
\sum_{F} h_{\tau}^{-1}\left\|w_{F}\right\|_{L^{2}(F)}^{2} \leq C\|\phi\|_{H}^{2} .
$$

Thus,

$$
\begin{aligned}
\left\|\phi_{h}\right\|_{H}^{2} & \leq C\left(\left\|I_{h} \phi\right\|_{H}^{2}+\left\|\phi_{\mathbb{F}}\right\|_{H}^{2}+\left\|\phi_{E}\right\|_{H}^{2}\right) \\
& \leq C\left(\|\phi\|_{H}^{2}+\sum_{\tau} h_{\tau}^{-2}\left\|w_{\tau}\right\|_{L^{2}(\tau)}^{2}\right) .
\end{aligned}
$$


Finally, by (6.5),

$$
\begin{aligned}
\sum_{\tau} h_{\tau}^{-2}\left\|w_{\tau}\right\|_{L^{2}(\tau)}^{2} & \leq C \sum_{\tau} h_{\tau}^{-2}\|\theta\|_{L^{2}(\tau)}^{2} \\
& \leq C \sum_{\tau} h_{\tau}^{-2}\left(\left\|\phi-I_{h} \phi\right\|_{L^{2}(\tau)}^{2}+\left\|\phi_{\mathbb{F}}\right\|_{L^{2}(\tau)}^{2}\right) \leq C\|\phi\|_{H^{1}(\Omega)}^{2} .
\end{aligned}
$$

This completes the proof of the lemma.

Remark 6.2. For this $\boldsymbol{X}_{h}$, the above constructions extend to the development of stable pairs for $H_{0}^{1}(\Omega),\left(H^{1}(\Omega)\right)^{3}$ and $\left(H_{0}^{1}(\Omega)\right)^{3}$ so that the analogs of Lemma 6.2 Lemma 6.3 and Lemma 6.4 hold.

Remark 6.3. In the case where linears and constants are mixed on different elements (in the definition of $\boldsymbol{X}_{h}$ ), we could modify the construction of $\boldsymbol{Y}_{h}$ and not include some of the functions in $B_{F}$. Using the full space $B_{F}$, even in this case, might be easier to implement.

Remark 6.4. It is clear that the constructions and analysis of this section could be extended to handle higher order approximation. For example, if $k$ was the maximum degree of the polynomial (in any component) on either side of $F$, one could use face bubble functions of degree $k+3$ in the neighboring tetrahedra. If $l$ was the maximum degree of the polynomial in any component on $\tau$, then one could use element bubble functions of degree $l+3$ on $\tau$.

Finally, in this section, we summarize the stability results for our two problems.

Theorem 6.1. Let $\boldsymbol{X}_{h}$ and $\boldsymbol{Y}_{h} \equiv \boldsymbol{V}_{h} \times H_{h}$ be constructed so that the conclusions of Lemma 6.1 and Lemma 6.3 are satisfied. Then for $\boldsymbol{u} \in \boldsymbol{X}_{h}$

$$
\|\boldsymbol{u}\|_{\mu^{-1}} \leq C \mu_{1}^{1 / 2} \sup _{(\boldsymbol{w}, \psi) \in \boldsymbol{Y}_{h}} \frac{b_{1}(\boldsymbol{u} ;(\boldsymbol{w}, \psi))}{\|(\boldsymbol{w}, \psi)\|_{\boldsymbol{Y}_{1}}} .
$$

Proof. Using (4.4), the theorem follows from Lemmas 6.1 and 6.3 .

Theorem 6.2. Let $\boldsymbol{X}_{h}$ and $\boldsymbol{Y}_{h} \equiv \boldsymbol{V}_{h} \times H_{h}$ be constructed so that the conclusions of Lemma 6.2 and Lemma 6.4 are satisfied. Then for $\boldsymbol{u} \in \boldsymbol{X}_{h}$

$$
\|\boldsymbol{u}\|_{\mu^{-1}} \leq C \mu_{1}^{1 / 2} \sup _{(\boldsymbol{w}, \psi) \in \boldsymbol{Y}_{h}} \frac{b_{2}(\boldsymbol{u} ;(\boldsymbol{w}, \psi))}{\|(\boldsymbol{w}, \psi)\|_{\boldsymbol{Y}_{2}}} .
$$

Proof. Using (5.3), the theorem follows from Lemmas 6.2 and 6.4 .

Remark 6.5. The discretizations implied by the above theorems are stable in $\left(L^{2}(\Omega)\right)^{3}$ and yield first order convergence when the solution is in $\left(H^{1}(\Omega)\right)^{3}$. By interpolation, we have $h^{s}$ convergence when the solution is in $\left(H^{s}(\Omega)\right)^{3}$ for any $s$ in $[0,1]$.

It is possible to get a stable approximation without the introduction of bubble functions (by form modification). For simplicity, we consider the case of Problem 1 and set $\boldsymbol{X}_{h}$ to be the space of piecewise constant vector functions. We define the discrete test spaces $\boldsymbol{V}_{h}$ and $H_{h}$ to be the piecewise linear vector or scalar functions defined on the same mesh satisfying the appropriate boundary conditions. These are the same spaces as those used above but without the bubble function enrichments. 
To get a stable approximation, we strengthen the form. We start from the inequality (4.7):

$$
C_{0} \mu_{1}^{-1}\|\boldsymbol{u}\|_{\mu^{-1}}^{2} \leq\left\|\operatorname{curl}_{\mu^{-1}} \boldsymbol{u}\right\|_{\boldsymbol{V}_{1}^{*}}^{2}+\left\|\operatorname{div}_{1} \boldsymbol{u}\right\|_{H_{1}^{*}}^{2} .
$$

We first consider the second term above. For $\boldsymbol{u}_{h} \in \boldsymbol{X}_{h}$ and $\phi_{h} \in H_{h}$,

$$
\left\|\operatorname{div}_{1} \boldsymbol{u}_{h}\right\|_{H_{1}^{*}}^{2} \leq C \sup _{\phi \in H_{1}}\left[\frac{\left(\boldsymbol{u}_{h}, \nabla\left(\phi-\phi_{h}\right)\right)^{2}}{\|\phi\|_{H_{1}}^{2}}+\frac{\left(\boldsymbol{u}_{h}, \nabla \phi_{h}\right)^{2}}{\|\phi\|_{H_{1}}^{2}}\right] .
$$

Let $\operatorname{div}_{1}^{h}: \boldsymbol{X}_{h} \mapsto H_{h}$ be defined by

$$
\left(\operatorname{div}_{1}^{h} \boldsymbol{y}_{h}, \theta_{h}\right)=-\left(\boldsymbol{y}_{h}, \nabla \theta_{h}\right) \text { for all } \theta_{h} \in H_{h} .
$$

Taking $\phi_{h}$ satisfying (6.5) in (6.15) and element by element integration by parts give

$$
\begin{aligned}
\left\|\operatorname{div}_{1} \boldsymbol{u}_{h}\right\|_{H_{1}^{*}}^{2} & \leq C \sup _{\phi_{h} \in H_{h}} \frac{\left(\boldsymbol{u}_{h}, \nabla \phi_{h}\right)^{2}}{\left\|\phi_{h}\right\|_{H_{1}}^{2}}+C \sum_{F} h_{F}\left\|\left[\boldsymbol{u}_{h} \cdot \boldsymbol{n}\right]\right\|_{L^{2}(F)}^{2} \\
& =C\left\|\operatorname{div}_{1}^{h} \boldsymbol{u}_{h}\right\|_{H_{h}^{*}}^{2}+C \sum_{F} h_{F}\left\|\left[\boldsymbol{u}_{h} \cdot \boldsymbol{n}\right]\right\|_{L^{2}(F)}^{2} .
\end{aligned}
$$

Here the sum is taken over all faces $F$ of the mesh, $h_{F}$ is the diameter of the face and $[\cdot]$ denotes the jump across the face. We used the inequality

$$
\left\|\phi-\phi_{h}\right\|_{L^{2}(F)} \leq C h_{F}^{1 / 2}\|\phi\|_{H^{1}\left(\tau_{F}\right)}
$$

which follows easily from (6.5). Here $\tau_{F}$ denotes the union of the tetrahedra which have $F$ as a face. Similarly, the first term of (6.14) is bounded by

$$
\left\|\operatorname{curl}_{\mu^{-1}} \boldsymbol{u}_{h}\right\|_{\boldsymbol{V}_{1}^{*}}^{2} \leq C\left\|\operatorname{curl}_{\mu^{-1}}^{h} \boldsymbol{u}_{h}\right\|_{\boldsymbol{V}_{h}^{*}}+C \sum_{F} h_{F}\left\|\left[\mu^{-1} \boldsymbol{u}_{h} \times \boldsymbol{n}\right]\right\|_{\left(L^{2}(F)\right)^{3}}^{2}
$$

with the analogous definition of $\operatorname{curl}_{\mu^{-1}}^{h}: \boldsymbol{X}_{h} \rightarrow \boldsymbol{V}_{h}^{*}$. Combining the above gives

$$
\left\|\boldsymbol{u}_{h}\right\|_{\mu^{-1}}^{2} \leq C A_{h}\left(\boldsymbol{u}_{h}, \boldsymbol{u}_{h}\right) \text { for all } \boldsymbol{u}_{h} \in \boldsymbol{X}_{h}
$$

where $A_{h}(\cdot, \cdot)$ is defined by

$$
\begin{aligned}
& A_{h}\left(\boldsymbol{x}_{h}, \boldsymbol{y}_{h}\right)=\left\langle\operatorname{curl}_{\mu^{-1}}^{h} \boldsymbol{x}_{h}, \widetilde{T}_{\boldsymbol{V}_{h}} \operatorname{curl}_{\mu^{-1}}^{h} \boldsymbol{y}_{h}\right\rangle+\left\langle\operatorname{div}_{1}^{h} \boldsymbol{x}_{h}, \widetilde{T}_{H_{h}} \operatorname{div}_{1}^{h} \boldsymbol{y}_{h}\right\rangle \\
& \quad+\sum_{F} h_{F}\left\{\left(\left[\mu^{-1} \boldsymbol{x}_{h} \times \boldsymbol{n}\right],\left[\mu^{-1} \boldsymbol{y}_{h} \times \boldsymbol{n}\right]\right)_{\left(L^{2}(F)\right)^{3}}+\left(\left[\boldsymbol{x}_{h} \cdot \boldsymbol{n}\right],\left[\boldsymbol{y}_{h} \cdot \boldsymbol{n}\right]\right)_{L^{2}(F)}\right\}
\end{aligned}
$$

The corresponding discretization now reads, find $\boldsymbol{x}_{h} \in \boldsymbol{X}_{h}$ satisfying

$$
A_{h}\left(\boldsymbol{x}_{h}, \boldsymbol{y}_{h}\right)=\left\langle\boldsymbol{j}, \widetilde{T}_{\boldsymbol{V}_{h}} \operatorname{curl}_{\mu^{-1}}^{h} \boldsymbol{y}_{h}\right\rangle+\left\langle l, \widetilde{T}_{H_{h}} \operatorname{div}_{1}^{h} \boldsymbol{y}_{h}\right\rangle, \quad \text { for all } \boldsymbol{y}_{h} \in \boldsymbol{X}_{h} .
$$

To analyze the error, we proceed as follows. For $\boldsymbol{x}$ in $\boldsymbol{X}$, we consider the map $\boldsymbol{x} \rightarrow \boldsymbol{x}_{h}$ where $\boldsymbol{x}_{h}$ is defined by (6.16) with $\boldsymbol{j}=\operatorname{curl}_{\mu^{-1}} \boldsymbol{x}$ and $l=\operatorname{div}_{1} \boldsymbol{x}$. By Theorem 4.1,

$$
\begin{aligned}
\left\|\boldsymbol{x}_{h}\right\|^{2} \leq & C A_{h}\left(\boldsymbol{x}_{h}, \boldsymbol{x}_{h}\right)=C\left\{\left\langle\boldsymbol{j}, \widetilde{T}_{\boldsymbol{V}_{h}} \operatorname{curl}_{\mu^{-1}}^{h} \boldsymbol{x}_{h}\right\rangle+\left\langle l, \widetilde{T}_{H_{h}} \operatorname{div}_{1}^{h} \boldsymbol{x}_{h}\right\rangle\right\} \\
\leq & \leq\|\boldsymbol{x}\|\left\|\boldsymbol{x}_{h}\right\| .
\end{aligned}
$$

By the triangle inequality,

$$
\left\|\boldsymbol{x}-\boldsymbol{x}_{h}\right\| \leq C\|\boldsymbol{x}\|, \quad \text { for all } \boldsymbol{x} \in \boldsymbol{X} .
$$


Partition $\Omega$ into subdomains $\Omega=\sum \Omega_{i}$ where $\mu$ is constant when restricted to $\Omega_{i}$ and define the space $\widetilde{\boldsymbol{H}}^{1}(\Omega)$ to be the functions which are in $\left(H^{1}\left(\Omega_{i}\right)\right)^{3}$ for each $i$ and satisfy the jump conditions

$$
[\boldsymbol{x} \cdot \boldsymbol{n}]=0 \text { and }\left[\mu^{-1} \boldsymbol{x} \times \boldsymbol{n}\right]=0
$$

on the interfaces between subdomains. Let $\boldsymbol{x}$ be in $\widetilde{\boldsymbol{H}}^{1}(\Omega)$. Then since $\mu$ is constant in $\Omega_{i}, \boldsymbol{x}$ satisfies the jump conditions (6.17) on all faces $\{F\}$.

As in the proof of Theorem 2.3, for any $\zeta \in \boldsymbol{X}_{h}$,

$$
\begin{aligned}
\left\|\boldsymbol{x}_{h}-\zeta\right\|_{\boldsymbol{X}}^{2} \leq & C A_{h}\left(\boldsymbol{x}_{h}-\zeta, \boldsymbol{x}_{h}-\zeta\right) \\
=C & \left\{\left\langle\operatorname{curl}_{\mu^{-1}}(\boldsymbol{x}-\zeta), \tilde{T}_{\boldsymbol{V}_{h}} \operatorname{curl}_{\mu^{-1}}^{h}\left(\boldsymbol{x}_{h}-\zeta\right)\right\rangle\right. \\
& +\left\langle\operatorname{div}_{1}(\boldsymbol{x}-\zeta), \tilde{T}_{H_{h}} \operatorname{div}_{1}^{h}\left(\boldsymbol{x}_{h}-\zeta\right)\right\rangle \\
& +\sum_{F} h_{F}\left[\left(\left[\mu^{-1}(\boldsymbol{x}-\zeta) \times \boldsymbol{n}\right],\left[\mu^{-1}\left(\boldsymbol{x}_{h}-\zeta\right) \times \boldsymbol{n}\right]\right)_{F}\right. \\
& \left.\left.+\left([(\boldsymbol{x}-\zeta) \cdot \boldsymbol{n}],\left[\left(\boldsymbol{x}_{h}-\zeta\right) \cdot \boldsymbol{n}\right]\right)_{F}\right]\right\} .
\end{aligned}
$$

It easily follows that

$$
\left\|\boldsymbol{x}-\boldsymbol{x}_{h}\right\| \leq C h\|\boldsymbol{x}\|_{\left(H^{1}(\Omega)\right)^{3}},
$$

for all $\boldsymbol{x} \in \widetilde{\boldsymbol{H}}^{1}(\Omega)$.

Let $\widetilde{\boldsymbol{H}}_{0}^{1}(\Omega)$ denote the subspace of functions in $\left(H^{1}(\Omega)\right)^{3}$ which vanish on the boundaries of all of the subdomains. Clearly $\widetilde{\boldsymbol{H}}_{0}^{1}(\Omega)$ is continuously imbedded in $\widetilde{\boldsymbol{H}}^{1}(\Omega)$. Now $H^{s}\left(\Omega_{i}\right)=H_{0}^{s}\left(\Omega_{i}\right)$ for $s<1 / 2$. Moreover, since extension by zero (cf. Theorem 1.4.4.5 of [17]) is continuous on $H^{s}\left(\Omega_{i}\right), H^{s}(\Omega)=\sum H^{s}\left(\Omega_{i}\right)$. It follows that for $s<1 / 2,\left(H^{s}(\Omega)\right)^{3}=\widetilde{\boldsymbol{H}}_{0}^{s}(\Omega)$ is continuously imbedded in $\widetilde{\boldsymbol{H}}^{s}(\Omega)$, the space obtained by interpolating between $\widetilde{\boldsymbol{H}}^{1}(\Omega)$ and $\left(L^{2}(\Omega)\right)^{3}$. Thus by interpolation, for $s<1 / 2$,

$$
\left\|\boldsymbol{x}-\boldsymbol{x}_{h}\right\| \leq C h^{s}\|\boldsymbol{x}\|_{\left(H^{s}(\Omega)\right)^{3}}, \quad \text { for all } \boldsymbol{x} \in\left(H^{s}(\Omega)\right)^{3} .
$$

Remark 6.6. If $\boldsymbol{x}$ is the solution of (4.6) and $\left.\boldsymbol{x}\right|_{\Omega_{i}}$ is in $\left(H^{s}\left(\Omega_{i}\right)\right)^{3}$ with $s>1 / 2$, then one would conjecture that $\boldsymbol{x} \in \widetilde{\boldsymbol{H}}^{s}(\Omega)$. By interpolation, both methods (with and without bubble functions) satisfy the error estimate

$$
\left\|\boldsymbol{x}-\boldsymbol{x}_{h}\right\| \leq C h^{s}\|\boldsymbol{x}\|_{\widetilde{\boldsymbol{H}}^{s}(\Omega)}, \quad \text { for all } \boldsymbol{x} \in \widetilde{\boldsymbol{H}}^{s}(\Omega) .
$$

\section{NUMERICAL EXPERIMENTS}

In this section, we report the results of numerical experiments which illustrate the theory of the previous sections. For ease of implementation, we report computations on two dimensional problems. Specifically, for a polygonal domain $\Omega$, we consider the problem: Find $\boldsymbol{h} \in \boldsymbol{X} \equiv\left(L^{2}(\Omega)\right)^{2}$ satisfying

$$
\begin{aligned}
\operatorname{rot} \boldsymbol{h} & =j \text { in } \Omega, \\
\nabla \cdot \mu \boldsymbol{h} & =g \text { in } \Omega, \\
(\mu \boldsymbol{h}) \cdot \boldsymbol{n} & =\sigma \text { on } \Gamma .
\end{aligned}
$$

In the above, $\operatorname{rot} \phi=\frac{\partial \phi_{2}}{\partial x_{1}}-\frac{\partial \phi_{1}}{\partial x_{2}}$ denotes the scalar curl. 
For this problem, both test spaces are scalar. In fact, we take $\boldsymbol{Y}=V \times H$ where $V \equiv H_{0}^{1}(\Omega)$ and $H=H^{1}(\Omega)$. Analogous to (4.3), we have

$$
\begin{aligned}
b_{1}(\boldsymbol{h} ; w, \phi) & \equiv(\boldsymbol{h}, \nabla \times w)+(\mu \boldsymbol{h}, \nabla \phi) \\
& =\langle j, w\rangle+(\sigma, \phi)_{\Gamma}-(g, \phi) \equiv\langle l ; w, \phi\rangle,
\end{aligned}
$$

for all $(w, \phi) \in \boldsymbol{Y}$. Here $\nabla \times \phi=\left(\frac{\partial \phi}{\partial x_{2}},-\frac{\partial \phi}{\partial x_{1}}\right)$ denotes the vector curl. The following result is a slight modification of Theorem 3.2 of [15]. A simple proof in the case of a simply connected domain is given below. We present it here since the two dimensional case differs from that of the three dimensional case in that the resulting norm equivalences are independent of $\mu$.

Lemma 7.1. For each function $\boldsymbol{u} \in\left(L^{2}(\Omega)\right)^{2}$ there exists a unique $(\cdot, \cdot)_{\mu}$-orthogonal decomposition

$$
\boldsymbol{u}=\mu^{-1} \nabla \times w+\nabla \phi \text { with }(w, \phi) \in \boldsymbol{Y} .
$$

Proof. We note that for any $(w, \phi) \in \boldsymbol{Y}$,

$$
\left(\mu^{-1} \nabla \times w, \nabla \phi\right)_{\mu}=(\nabla \times w, \nabla \phi)=\left(w, \nabla \phi \cdot \boldsymbol{n}^{\perp}\right)_{\Gamma}=0 .
$$

We next observe that $\mu^{-1} \nabla \times V+\nabla H=\left(L^{2}(\Omega)\right)^{2}$. Indeed if $\boldsymbol{u} \in\left(L^{2}(\Omega)\right)^{2}$ satisfies

$$
\left(\mu^{-1} \nabla \times w, \boldsymbol{u}\right)_{\mu}=0 \quad \text { for all } w \in V
$$

then $\operatorname{rot} \boldsymbol{u}=0$ and by Theorem 2.9 of [15], $\boldsymbol{u}=\nabla \phi$ for some $\phi \in H$.

Finally, the functions $[w, \phi] \in \boldsymbol{Y}$ satisfying (17.1) are the unique solutions to the problems

$$
(\nabla \times w, \nabla \times \theta)_{\mu^{-1}}=(\boldsymbol{u}, \nabla \times \theta) \text { for all } \theta \in V
$$

and

$$
(\nabla \phi, \nabla \zeta)_{\mu}=(\boldsymbol{u}, \nabla \zeta)_{\mu} \quad \text { for all } \zeta \in H .
$$

This completes the proof of the lemma.

It follows from the above lemma that for all $\boldsymbol{u} \in\left(L^{2}(\Omega)\right)^{2}$

$$
\|\boldsymbol{u}\|_{\mu}^{2}=\sup _{w \in V} \frac{(\boldsymbol{u}, \nabla \times w)^{2}}{\|\nabla \times w\|_{\mu^{-1}}^{2}}+\sup _{\phi \in H} \frac{(\mu \boldsymbol{u}, \nabla \phi)^{2}}{\|\nabla \phi\|_{\mu}^{2}} .
$$

Thus, it is natural to use the weighted inner products $(\cdot, \cdot)_{\mu^{-1}}$ and $(\cdot, \cdot)_{\mu}$ for $V$ and $H$, respectively. In this case, the iterative convergence rate is independent of $\mu$ provided that the preconditioners $\widetilde{T}_{Y}$ and $\widetilde{T}_{H}$ are uniformly equivalent to $T_{Y}$ and $T_{H}$, respectively, independently of $\mu$.

In all of our examples, we partition the domain $\Omega$ into a shape regular mesh. For $\boldsymbol{X}_{h}$, we used piecewise constant vector approximation. The spaces $V_{h}$ and $H_{h}$ are defined as piecewise linear and continuous (vanishing on $\partial \Omega$ in the case of $V_{h}$ ) enriched by edge bubble functions as discussed in Section 6. The preconditioners for $T_{Y}$ and $T_{H}$ were defined by a multiplicative two level algorithm involving GaussSiedel smoothing on the bubble nodes and a direct solve on the subspace of piecewise linears.

The first problem is an application involving a smooth solution. We let $\Omega$ be the unit square and take $\mu=1, j=0, g=\cos (\pi x) \cos (\pi y)$ and $\sigma=0$. This problem has solution

$$
\boldsymbol{h}=(\sin (\pi x) \cos (\pi y), \cos (\pi x) \sin (\pi y)) /(2 \pi) .
$$


TABLE 7.1. Numerical results with smooth solution (17.2).

\begin{tabular}{|c|c|c|c|c|}
\hline$h$ & $L^{2}$-error & ratio & \#iterations & \#unknowns \\
\hline $1 / 8$ & 0.576961 & & 6 & 256 \\
\hline $1 / 16$ & 0.290813 & 1.98396 & 6 & 1024 \\
\hline $1 / 32$ & 0.145741 & 1.99541 & 6 & 4096 \\
\hline $1 / 64$ & 0.072897 & 1.99926 & 5 & 16384 \\
\hline $1 / 128$ & 0.036451 & 1.99984 & 5 & 65536 \\
\hline $1 / 256$ & 0.018226 & 1.99997 & 4 & 262144 \\
\hline
\end{tabular}

TABLE 7.2. Numerical results for the L-shaped example.

\begin{tabular}{|c|c|c|c|c|}
\hline$h$ & $L^{2}$-error & ratio & \#iterations & \#unknowns \\
\hline 0.176777 & 0.223524 & & 11 & 512 \\
\hline 0.0883883 & 0.143219 & 1.56072 & 11 & 2048 \\
\hline 0.0441942 & 0.091108 & 1.57196 & 11 & 8192 \\
\hline 0.0220971 & 0.057727 & 1.57826 & 11 & 32768 \\
\hline 0.0110485 & 0.036492 & 1.58188 & 11 & 131072 \\
\hline 0.00552427 & 0.023038 & 1.58483 & 11 & 524288 \\
\hline
\end{tabular}

TABLE 7.3. Numerical results for the jumping coefficient problem.

\begin{tabular}{|c|c|c|c|}
\hline$h_{\min }$ & $h_{\max }$ & \#iterations & \# unknowns \\
\hline 0.0316111 & 0.316228 & 9 & 152 \\
0.0158055 & 0.158114 & 11 & 608 \\
0.0079027 & 0.079056 & 12 & 2432 \\
0.0039513 & 0.039528 & 11 & 9728 \\
0.0019756 & 0.019764 & 13 & 38912 \\
\hline
\end{tabular}

We use a regular mesh of triangles obtained by first partitioning the square into $n \times n$ equal smaller squares and dividing each smaller square into two by the positive sloping diagonal.

The numerical results are given in Table 7.1. The error behavior in $\left(L^{2}(\Omega)\right)^{2}$ clearly illustrates the expected first order convergence rate. Note that the number of iterations required to reduce the residual by a factor of $10^{-6}$ remains bounded independently of the number of unknowns.

For the second example, we consider a problem on the L-shaped domain consisting of the square $[-1,1]^{2}$ with the area in the fourth quadrant removed. Because of the re-entrant corner, solutions of problems on this domain are not smooth in general. We take a problem which illustrates the typical singularity and we take $j, g$, and $\sigma$ so that the solution is given (in polar coordinates) by $\boldsymbol{h}=\operatorname{grad}\left(r^{\beta} \cos (\beta \theta)\right)$ with $\beta=2 / 3$. The function $\boldsymbol{h}$ is only in $\left(H^{s}(\Omega)\right)^{2}$ for $s<2 / 3$. In this case, we expect that a mesh reduction of a factor of two should result in an error reduction of $2^{2 / 3} \approx 1.587$. This is clearly illustrated by the convergence results in Table 7.2 Again, we see that the number of iterations remains bounded as the mesh size is decreased. 

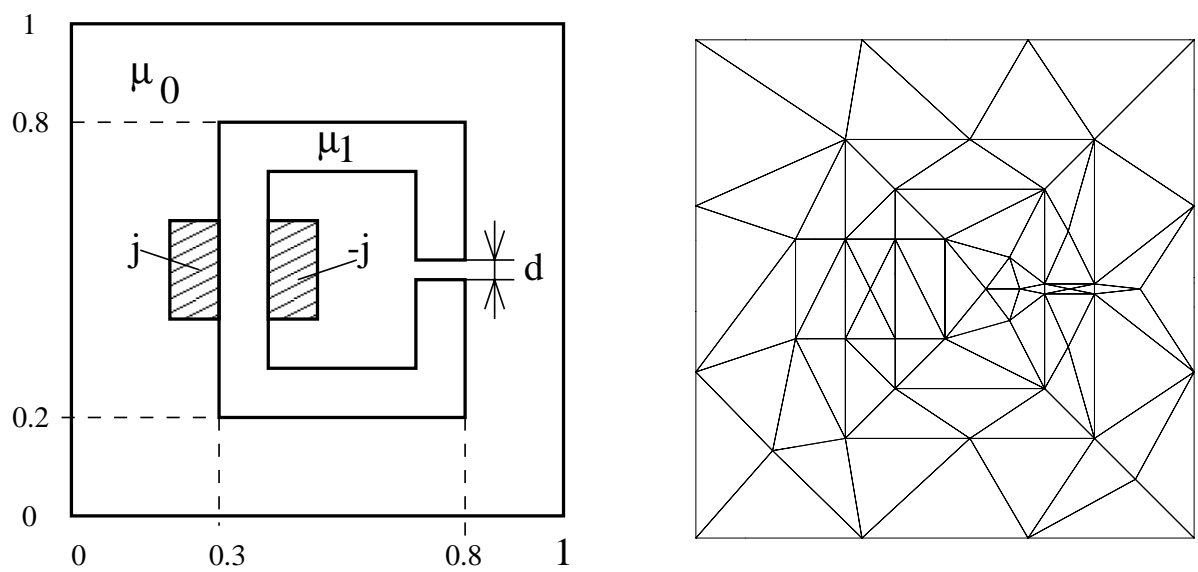

Figure 1. A jumping coefficient example and coarse mesh

We finally report numerical results for a problem with jumps in coefficients. We consider the geometry given in Figure 1 This consists of a iron segment with fixed magnetic permeability $\mu_{1}=1000$ surrounded by an air region with permeability $\mu_{0}=1$. A uniform current of $j$ and $-j$ (shaded regions) is applied in the $z$ direction. There is also a small air gap of size $d=.01$. We modeled a perfectly conducting outer boundary and set $\boldsymbol{b} \cdot \boldsymbol{n}=0$ there. For this problem, we do not report the error behavior as the analytic solution is not available. Our goal was to illustrate the iterative convergence rate. The numerical experiments reported in Table $7.3 \mathrm{show}$ that even though there are large jumps in the permeability, the iterative process still converges in relatively few iterations. It also shows that the method performs well even in the case of a fairly anisotropic mesh (see Figure 1).

\section{ACKnowledgment}

We would like to thank T. Kolev for providing the computations reported in Section 7

\section{REFERENCES}

[1] C. Amrouche, C. Bernardi, M. Dauge, and V. Girault. Vector potentials in three-dimensional non-smooth domains. Math. Methods Appl. Sci., 21(9):823-864, 1998. MR 99e:35037

[2] G. Auchmuty and J. C. Alexander. $L^{2}$ well-posedness of planar div-curl systems. Arch. Ration. Mech. Anal., 160(2):91-134, 2001. MR 2002i:35022

[3] A. Aziz and I. Babuška. Part I, survey lectures on the mathematical foundations of the finite element method. In A. Aziz, editor, The Mathematical Foundations of the Finite Element Method with Applications to Partial Differential Equations, pages 1-362, New York, NY, 1972. Academic Press. MR 54:9111

[4] A. Bossavit. Computational electromagnetism. Academic Press Inc., San Diego, CA, 1998. Variational formulations, complementarity, edge elements. MR 99m:78001

[5] J. H. Bramble, R. D. Lazarov, and J. E. Pasciak. Least-squares for second order elliptic problems. Comput. Meth. Appl. Mech. Engrg., 152:195-210, 1998. MR 99a:65145

[6] J. H. Bramble, R. D. Lazarov, and J. E. Pasciak. A least-squares approximation of problems in linear elasticity based on a discrete minus one inner product. Comput. Meth. Appl. Mech. Engrg., 191:520-543, 2001. 
[7] F. Brezzi and M. Fortin. Mixed and Hybrid Finite Element Methods. Springer-Verlag, New York, 1991. MR 92d:65187

[8] C. L. Chang. Finite element approximation for grad-div type of systems in the plane. SIAM J. Numerical Analysis, 29:590-601, 1992. MR 92k:65159

[9] P. Clément. Approximation by finite element functions using local regularization. Rev. Française Automat. Informat. Recherche Opérationnelle Sér. Rouge Anal. Numér., 9(R-2):77-84, 1975. MR 53:4569

[10] M. Costabel and M. Dauge. Singularities of electromagnetic fields in polyhedral domains. Arch. Ration. Mech. Anal., 151(3):221-276, 2000. MR 2002c:78005

[11] M. Costabel, M. Dauge, and D. Martin. Numerical investigation of a boundary penalization method for Maxwell equations. 1999. Preprint.

[12] M. Costabel, M. Dauge, and D. Martin. Weighted regularization of Maxwell equations in polyhedral domains. 2001. Preprint.

[13] M. Costabel, M. Dauge, and S. Nicaise. Singularities of Maxwell interface problems. M2AN Math. Model. Numer. Anal., 33(3):627-649, 1999. MR 2001g:78005

[14] L. Demkowicz and L. Vardapetyan. Modeling of electromagnetic absorption/scattering problems using $h p$-adaptive finite elements. Comput. Methods Appl. Mech. Engrg., 152(1-2):103124, 1998. Symposium on Advances in Computational Mechanics, Vol. 5 (Austin, TX, 1997). MR 99b:78003

[15] V. Girault and P. Raviart. Finite Element Approximation of the Navier-Stokes Equations. Lecture Notes in Math. \# 749, Springer-Verlag, New York, 1981. MR 83b:65122

[16] J. Gopalakrishnan and J. E. Pasciak. Overlapping Schwarz preconditioners for indefinite time harmonic Maxwell equations. Math. Comp., 72(241):1-15, 2003.

[17] P. Grisvard. Elliptic Problems in Nonsmooth Domains. Pitman, Boston, 1985. MR 86m:35044

[18] R. Hiptmair. Multigrid method for Maxwell's equations. SIAM J. Numer. Anal., 36(1):204225, 1999. MR 99j:65229

[19] R. Hiptmair. Analysis of multilevel methods for eddy current problems. Math. Comp., 72(243):1281-1303, 2003.

[20] R. Hiptmair and A. Toselli. Overlapping Schwarz methods for vector valued elliptic problems in three dimensions. In Parallel solution of PDEs, IMA Volumes in Mathematics and its Applications. Springer-Verlag, Berlin, 1998. MR 2002d:65123

[21] J. C. Nedelec. Mixed finite elements in $\mathbf{R}^{3}$. Numer. Math., 35:315-341, 1980. MR 81k:65125

[22] J. C. Nedelec. A new family of mixed finite elements in $\mathbf{R}^{3}$. Numer. Math., 50:57-81, 1986. MR 88e:65145

[23] R. A. Nicolaides. Direct discretization of planar div-curl problems. SIAM J. Numer. Anal., 29(1):32-56, 1992. MR 93b:65176

[24] R. A. Nicolaides and X. Wu. Covolume solutions of three-dimensional div-curl equations. SIAM J. Numer. Anal., 34(6):2195-2203, 1997. MR 98f:65096

[25] J. Pasciak and J. Zhao. Overlapping Schwarz methods in $H$ (curl) on polyhedral domains. $J$. Numer. Math., 10:221-234, 2002.

[26] I. Perugia, D. Schötzau, and P. Monk. Stabilized interior penalty methods for the timeharmonic Maxwell equations, Comput. Methods Appl. Mech. Engrg. 191(41-42):4675-4697, 2002.

[27] S. Reitzinger and J. Schöberl. An algebraic multigrid method for finite element discretizations with edge elements. Numer. Linear Algebra Appl., 9(3):223-238, 2002. MR 2003f:78033

[28] A. Toselli. Overlapping Schwarz methods for Maxwell's equations in three dimensions. Numer. Math., 86(4):733-752, 2000. MR 2001h:65137

Department of Mathematics, Texas A\&M University, College Station, Texas 778433368

E-mail address: bramble@math.tamu.edu

Department of Mathematics, Texas A\&M University, College Station, Texas 778433368

E-mail address: pasciak@math.tamu.edu 